\title{
"Syn Effect" in Nucleophilic Addition of Amines to 1,3-Dienyl Sulfone and Ethyl (E)-2,4-Pentadienoate
}

\author{
Masao Yamazaki, Samar Kumar Guha, Yutaka Ukaji,* and Katsuhiko Inomata* \\ Division of Material Sciences, Graduate School of Natural Science and Technology, \\ Kanazawa University, Kakuma, Kanazawa 920-1192 \\ Received December 17, 2007; E-mail: inomata@cacheibm.s.kanazawa-u.ac.jp
}

\begin{abstract}
The stereochemistry of nucleophilic addition of amines to $(E)$-1-tosyl-1,3-butadiene was investigated. The Z/E ratios of the resulting allylic sulfones varied with amines, solvents, temperature, and concentration. When diethylamine was reacted in low concentration at high temperature, the corresponding sterically unfavorable (Z)-4-amino-2-butenyl sulfone was preferentially obtained. The stereochemistry of nucleophilic addition of amines to ethyl $(E)$-2,4-pentadienoate, which possesses an ester group as a conjugated electron-withdrawing group instead of a $p$-toluenesulfonyl (Ts) group, was also found to realize similar high $\mathrm{Z}$ selectivity. The predominant formation of $\mathrm{Z}$ isomers in both cases was rationalized by a "syn effect," which might be mainly due to $\mathrm{n} / \sigma \rightarrow \pi^{*}$ interaction and/or $6 \pi$-electron homoaromaticity.
\end{abstract}

Allylic sulfones are versatile synthetic intermediates in organic synthesis. ${ }^{1}$ During the course of studies on the preparation of allylic sulfones, ${ }^{2}$ we investigated the stereochemistry of isomerization of $\alpha$-unsubstituted $(E)$-vinylic sulfones to the corresponding allylic sulfones in the presence of a base and found that the sterically unfavorable $(Z)$-allylic sulfones were predominantly formed. ${ }^{3}$ This result was rationalized by a "syn effect," ${ }^{4}$ which is primarily caused by $\sigma \rightarrow \pi^{*}$ interaction and/or $6 \pi$-electron homoaromaticity (Figure 1). ${ }^{5}$ In studies related to allylic sulfones, the predominant formation of (Z)-olefins, which could also be ascribed to the "syn effect," was found in the desulfonylation reaction of $\alpha, \alpha$-dialkylated $(E)$-allylic sulfones, ${ }^{5 \mathrm{a}}$ the isomerization of $(E)-\alpha$-fluorovinylic sulfones to the corresponding allylic sulfones under basic conditions ${ }^{5 b}$ and the desilylation reaction of $\gamma$-silylated allylic and vinylic sulfones. ${ }^{5 \mathrm{~d}}$ Furthermore, we revealed that the "syn effect" works also in the conversion of $(E)-\alpha, \beta$-unsaturated esters and aldehydes into the corresponding $\beta, \gamma$-unsaturated esters and silyl enol ethers, ${ }^{5 c, 5 \mathrm{e}}$ respectively, the elimination reaction of $(E)$-allylic acetates catalyzed by palladium under specific conditions utilizing a base ${ }^{5 \mathrm{f}}$ and the 1,4 -eliminative ring-opening reaction of $(E)$-1-propenyloxirane derivatives by treatment with metal amides. ${ }^{5 \mathrm{~g}}$

For the preparation of allylic sulfones, nucleophilic addition to dienyl sulfones is a useful method. A couple of reactions of conjugate addition to dienyl sulfones have been reported. ${ }^{6,7} \mathrm{~A}$ nucleophilic addition of aniline derivatives to $(E)$-1-tosyl-1,3butadiene (1) in the presence of $\mathrm{K}_{2} \mathrm{CO}_{3}$ afforded the corresponding $(E)$-allylic sulfones with good to complete stereoselectivity. ${ }^{6 a}$ Asymmetric conjugate addition of a $\beta$-ketoester to $(E)$-1-(benzenesulfonyl)-1,3-butadiene in the presence of $\mathrm{K}_{2} \mathrm{HPO}_{4}$ also gave the corresponding adduct with complete E selectivity. ${ }^{6 \mathrm{~b}}$ Interestingly, it has been reported that addition of a transition-metal reagent, lithium dibutylcuprate, to $(E)-1$ (allylsulfonyl)-1,3-butadiene gave a 65/35 mixture of $(Z)$ - and $(E)$-allylic sulfones. Furthermore, the addition of the same lithium dibutylcuprate to $\mathbf{1}$ was reported to give only (Z)-1-tosyl2 -octene in $21 \%$ yield..$^{6 c}$ However, both isomers were obtained in $96 \%$ total yield with $\mathrm{Z}$ preference $(\mathrm{Z} / \mathrm{E}=65 / 35)$ in our reexamination. This inconsistent result prompted us to investigate the stereochemistry of the nucleophilic addition of various nucleophiles. Among non-metallic compounds, amines showed various stereoselectivities depending on the kinds of amines and reaction conditions. Herein, we describe the results of the stereochemistry of the nucleophilic addition of amines to $(E)$-1-tosyl-1,3-butadiene $(\mathbf{1})^{8}$ and to ethyl $(E)$-2,4-pentadienoate (4).

\section{Results and Discussion}

First, the nucleophilic addition of various amines $\mathbf{2 a}-\mathbf{2} \mathbf{k}$ to
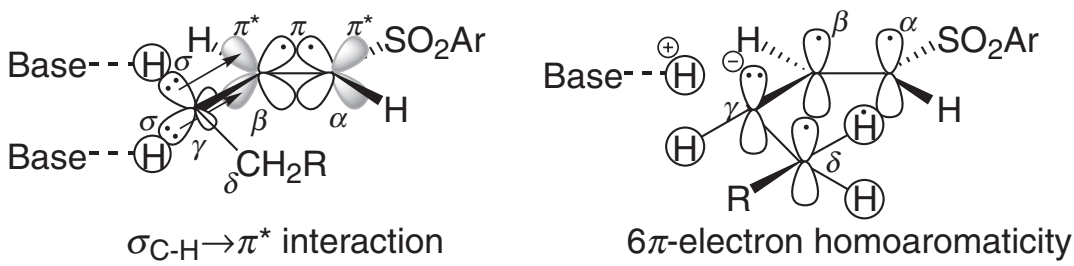

Figure 1. Proposed origin of the "syn effect" in the isomerization of $\alpha$-unsubstituted $(E)$-vinylic sulfones to the corresponding allylic sulfones. 
(E)-1-tosyl-1,3-butadiene (1) was carried out in THF at $25^{\circ} \mathrm{C}$ and the results are summarized in Table 1 . The $\mathrm{Z} / \mathrm{E}$ ratios of the resulting allylic sulfones $\mathbf{3 a}-\mathbf{3 k}$ varied depending on the kinds of amines. Primary amines such as propylamine and butylamine gave the corresponding $(E)$-allylic amines mainly (Entries 1 and 2). To the contrary, acyclic secondary amines preferentially formed (Z)-allylic amines (Entries 3-5 and 79 ) as opposed to $\mathrm{E}$ isomers. Especially, $n-\mathrm{Pr}_{2} \mathrm{NH}$ and $n-\mathrm{Bu}_{2} \mathrm{NH}$ showed relatively high $\mathrm{Z}$ preference although the reaction was sluggish (Entries 5 and 7). Addition of cyclic secondary amines gave almost equal amounts of $(Z)$ - and $(E)$-allylic amines (Entries 10 and 11).

Next, the stereochemistry of the nucleophilic addition of $\mathrm{Et}_{2} \mathrm{NH}$ (2d) to (E)-1-tosyl-1,3-butadiene (1) was examined in detail, paying attention to the effect of solvents, temperature,

Table 1. Stereochemistry of Nucleophilic Addition of Various Amines 2a-2k to (E)-1-Tosyl-1,3-butadiene (1) ${ }^{\text {a) }}$

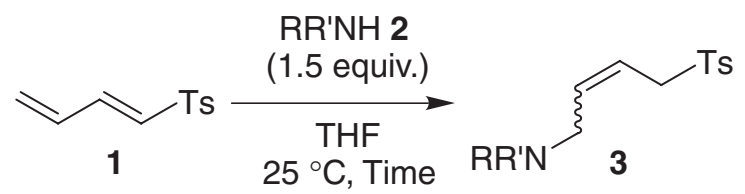

\begin{tabular}{ccccccc}
\hline Entry & $\mathrm{RR}^{\prime} \mathrm{NH} \mathrm{2}$ & & Time $/ \mathrm{h}$ & $\mathbf{1 / \mathbf { 3 } ^ { \mathrm { b } ) }}$ & Yield $/ \%^{\mathrm{c})}$ & $\mathrm{Z} / \mathrm{E}^{\mathrm{d})}$ \\
\hline $1^{\mathrm{e})}$ & $n-\mathrm{PrNH}_{2}$ & $\mathbf{a}$ & 72 & $11 / 89$ & 70 & $21 / 79$ \\
$2^{\mathrm{e})}$ & $n-\mathrm{BuNH}_{2}$ & $\mathbf{b}$ & 72 & $11 / 89$ & 71 & $23 / 77$ \\
3 & $\left.\mathrm{Me}_{2} \mathrm{NH}^{\mathrm{f}}\right)$ & $\mathbf{c}$ & 24 & $0 / 100$ & 91 & $60 / 40$ \\
4 & $\mathrm{Et}_{2} \mathrm{NH}$ & $\mathbf{d}$ & 72 & $19 / 81$ & 75 & $74 / 26$ \\
5 & $n-\mathrm{Pr}_{2} \mathrm{NH}$ & $\mathbf{e}$ & 72 & $52 / 48$ & 43 & $85 / 15$ \\
6 & $i-\mathrm{Pr}_{2} \mathrm{NH}$ & $\mathbf{f}$ & 72 & $100 / 0$ & - & - \\
7 & $n-\mathrm{Bu}_{2} \mathrm{NH}$ & $\mathbf{g}$ & 72 & $48 / 52$ & 46 & $87 / 13$ \\
8 & $n-\mathrm{Bu}(\mathrm{Me}) \mathrm{NH}$ & $\mathbf{h}$ & 72 & $0 / 100$ & 85 & $72 / 28$ \\
9 & $i$-Pr(Me)NH & $\mathbf{i}$ & 72 & $38 / 62$ & 58 & $80 / 20$ \\
10 & Pyrrolidine & $\mathbf{j}$ & 6 & $0 / 100$ & 83 & $44 / 56$ \\
11 & Piperidine & $\mathbf{k}$ & 12 & $0 / 100$ & 85 & $55 / 45$ \\
\hline
\end{tabular}

a) Concentration of amines 2 was $150 \mathrm{mM}\left(\mathrm{mmol} \mathrm{dm}^{-3}\right)$ in all cases. b) The ratios were determined based on the isolated yields. c) Isolated total yield of 3. d) The ratios were determined by $400 \mathrm{MHz}{ }^{1} \mathrm{HNMR}$ spectra. e) Formation of $\left(\mathrm{TsCH}_{2} \mathrm{CH}=\mathrm{CHCH}_{2}\right)_{2} \mathrm{NR}(\mathrm{R}=n$-Pr, $5 \% ; n-\mathrm{Bu}, 7 \%)$ was observed. f) A commercially available $2.0 \mathrm{M}\left(\mathrm{mol} \mathrm{dm}^{-3}\right)$ solution of $\mathrm{Me}_{2} \mathrm{NH}$ in THF was used. and concentration; the results are summarized in Tables 2 and 3 . Ethereal solvents generally afforded the $(Z)$-allylic amine preferentially, but less polar benzene and highly polar $\mathrm{CH}_{3} \mathrm{CN}$ and DMSO gave the $\mathrm{E}$ isomer mainly as shown in Table 2. It was found that polar and less bulky ethers, such as DME and THF, showed high $\mathrm{Z}$ selectivity (Entries 2 and 5). The time-course of the addition reaction checked by ${ }^{1} \mathrm{HNMR}$ in $\mathrm{THF}-d_{8}$ revealed that the $\mathrm{Z} / \mathrm{E}$ ratio was almost constant as the reaction proceeded. ${ }^{9}$ It is noteworthy that the $\mathrm{Z}$ selectivity was enhanced when the reaction was carried

Table 2. Stereochemistry of Nucleophilic Addition of $\mathrm{Et}_{2} \mathrm{NH}(\mathbf{2 d})$ to $(E)$-1-Tosyl-1,3-butadiene (1) in Various Solvents ${ }^{\text {a) }}$

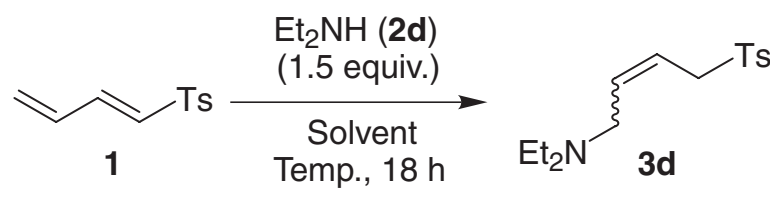

\begin{tabular}{|c|c|c|c|c|c|}
\hline Entry & Solvent & $\mathrm{Temp} /{ }^{\circ} \mathrm{C}$ & $1 / 3 d^{b)}$ & Yield $/ \%^{c)}$ & $\mathrm{Z} / \mathrm{E}^{\mathrm{d})}$ \\
\hline 1 & DME & 0 & $84 / 16$ & 16 & $67 / 33$ \\
\hline 2 & & 25 & $58 / 42$ & 40 & $82 / 18$ \\
\hline 3 & & 60 & $33 / 67$ & 61 & $88 / 12$ \\
\hline 4 & THF & 0 & $65 / 35$ & 28 & $52 / 48$ \\
\hline 5 & & 25 & $59 / 41$ & 38 & $78 / 22$ \\
\hline 6 & & 60 & $27 / 73$ & 64 & $86 / 14$ \\
\hline 7 & 1,4-Dioxane & 25 & $32 / 68$ & 61 & $64 / 36$ \\
\hline 8 & THP & 25 & $41 / 59$ & 48 & $61 / 39$ \\
\hline 9 & $\mathrm{Et}_{2} \mathrm{O}$ & 25 & $61 / 39$ & 35 & $31 / 69$ \\
\hline 10 & $t$-BuOMe & 25 & $52 / 48$ & 47 & $28 / 72$ \\
\hline 11 & Pyridine & 25 & $3 / 97$ & 73 & $71 / 29$ \\
\hline 12 & $N$-Methylmorpholine & 25 & $71 / 29$ & 26 & $63 / 37$ \\
\hline 13 & $N$-Methylpyrrolidine & 25 & $81 / 19$ & 15 & $53 / 47$ \\
\hline 14 & $\mathrm{CHCl}_{3}$ & 25 & $44 / 56$ & 56 & $44 / 56$ \\
\hline 15 & Tetrahydrothiophene & 25 & $0 / 100$ & 90 & $41 / 59$ \\
\hline 16 & Benzene & 25 & $27 / 73$ & 70 & $30 / 70$ \\
\hline 17 & $\mathrm{MeCN}$ & 25 & $0 / 100$ & 98 & $37 / 63$ \\
\hline 18 & DMSO & 25 & $0 / 100$ & 77 & $27 / 73$ \\
\hline
\end{tabular}

a) Concentration of $\mathbf{2 d}$ was $150 \mathrm{mM}$ in all cases. b) The ratios were determined based on the isolated yields. c) Isolated total yield of 3d. d) The ratios were determined by $400 \mathrm{MHz}$ ${ }^{1}$ H NMR spectra.

Table 3. Effect of Concentration on Nucleophilic Addition of $\mathrm{Et}_{2} \mathrm{NH}$ (2d) to (E)-1-Tosyl-1,3-butadiene (1)

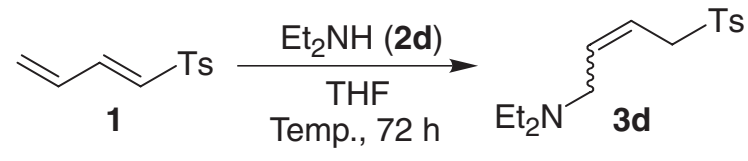

\begin{tabular}{|c|c|c|c|c|c|c|c|}
\hline \multirow{2}{*}{ Entry } & \multicolumn{2}{|c|}{ Concentrations } & \multirow{2}{*}{$2 d / 1$} & \multirow{2}{*}{ Temp $/{ }^{\circ} \mathrm{C}$} & \multirow{2}{*}{$\mathbf{1} / \mathbf{3} \mathbf{d}^{\mathrm{a})}$} & \multirow{2}{*}{ Yield/\%b) } & \multirow{2}{*}{$\mathrm{Z} / \mathrm{E}^{\mathrm{c})}$} \\
\hline & $1 / \mathrm{mM}$ & $2 \mathrm{~d} / \mathrm{mM}$ & & & & & \\
\hline 1 & 100 & 150 & 1.5 & 25 & $19 / 81$ & 75 & $74 / 26$ \\
\hline 2 & 50 & 75 & 1.5 & 25 & $51 / 49$ & 48 & $93 / 7$ \\
\hline 3 & 25 & 37.5 & 1.5 & 25 & $64 / 36$ & 33 & $96 / 4$ \\
\hline 4 & 25 & 37.5 & 1.5 & 60 & $38 / 62$ & 50 & $96 / 4$ \\
\hline 5 & 3.1 & 37.5 & 12.0 & 60 & $28 / 72$ & 72 & $98 / 2$ \\
\hline 6 & 10 & 15 & 1.5 & 25 & $86 / 14$ & 12 & $94 / 6$ \\
\hline
\end{tabular}

a) The ratios were determined based on the isolated yields. b) Isolated total yield of $\mathbf{3 d}$. c) The ratios were determined by $400 \mathrm{MHz}{ }^{1} \mathrm{H} \mathrm{NMR}$ spectra. 
Table 4. Stereochemistry of Nucleophilic Addition of $\mathrm{Et}_{2} \mathrm{NH}(\mathbf{2 d})$ to Ethyl $(E)-2,4-P e n t a d i e n o a t e ~(4)$ in Various Solvents ${ }^{\text {a) }}$

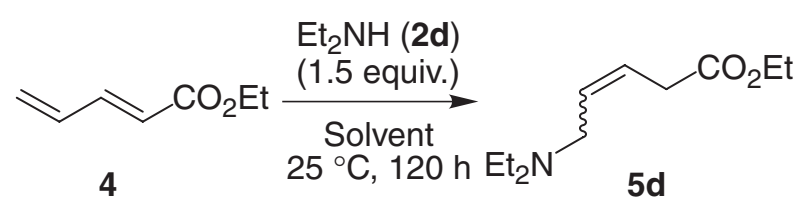

\begin{tabular}{|c|c|c|c|c|}
\hline Entry & Solvent & $4 / 5 d^{b)}$ & Yield $/ \%^{c)}$ & $\mathrm{Z} / \mathrm{E}^{\mathrm{d})}$ \\
\hline 1 & DME & $87 / 13$ & 9 & $85 / 15$ \\
\hline 2 & THF & $88 / 12$ & 10 & $86 / 14$ \\
\hline 3 & 1,4-Dioxane & $92 / 8$ & 6 & $69 / 31$ \\
\hline 4 & THP & $93 / 7$ & 5 & $69 / 31$ \\
\hline 5 & $\mathrm{Et}_{2} \mathrm{O}$ & $94 / 6$ & 4 & $42 / 58$ \\
\hline 6 & Pyridine & $58 / 42$ & 30 & $23 / 77^{\mathrm{e}}$ \\
\hline 7 & $\mathrm{CHCl}_{3}$ & $91 / 9$ & 7 & $34 / 66$ \\
\hline 8 & Benzene & $90 / 10$ & 7 & $49 / 51$ \\
\hline 9 & $\mathrm{MeCN}$ & $65 / 35$ & 34 & $17 / 83^{\mathrm{e}}$ \\
\hline 10 & $\mathrm{EtOH}$ & $79 / 21$ & 18 & $9 / 91$ \\
\hline
\end{tabular}

a) Concentration of $\mathrm{Et}_{2} \mathrm{NH}$ (2d) was $150 \mathrm{mM}$ in all cases. b) The ratios were determined based on the isolated yields. c) Isolated total yield of $\mathbf{5 d}$ based on $\mathbf{4}$. d) The ratios were determined by $400 \mathrm{MHz}{ }^{1} \mathrm{HNMR}$ spectra. ${ }^{11} \mathrm{e}$ ) Isomerization of $\mathrm{Z}$ isomer to $\mathrm{E}$ isomer was observed during the reaction.

out at higher temperature (Entries 1-6).

The effect of concentration is shown in Table 3. The lower concentration of $\mathrm{Et}_{2} \mathrm{NH}$ remarkably increased the $\mathrm{Z}$ selectivity of 3d (Entries 1-3 and 6), though the reaction became sluggish. Finally, using excess $\mathrm{Et}_{2} \mathrm{NH}$ at $60^{\circ} \mathrm{C},(Z)$-allylic sulfone 3d was obtained in $72 \%$ yield with the highest $\mathrm{Z}$ selectivity (Entry 5).

As we described above, unprecedented $\mathrm{Z}$ selectivity in the conjugate addition of amines to (E)-1-tosyl-1,3-butadiene (1) was discovered. This phenomenon was suspected to be specific to sulfonyl compounds. Therefore, nucleophilic addition of amines to ethyl (E)-2,4-pentadienoate (4), which possesses an ester group as a conjugated electron-withdrawing group instead of a $p$-toluenesulfonyl (Ts) group, was next carried out.

Conjugate 1,6-addition reactions to $(E)$-2,4-pentadienoate were reported to generally furnish the corresponding $(E)-3$ pentenoate. ${ }^{10}$ For example, addition of nitroalkanes in the presence of Amberlyst A 27 or a lithium salt of a bislactim ether afforded the exclusively E adducts. ${ }^{10 a, 10 b}$ Asymmetric conjugate addition of $\beta$-ketoesters to $(E)$-2,4-pentadienoate using an inorganic base in the presence of cinchona alkaloids also gave the corresponding adduct with complete E selectivity. ${ }^{6 b}$ Nickel-catalyzed addition of morpholine has been reported to give an $(E)$-allylic amine. ${ }^{10 \mathrm{c}}$ Addition of organocopper reagents afforded $(E)-\beta, \gamma$-unsaturated esters. ${ }^{10 \mathrm{~d}}$ Only the transitionmetal-catalyzed addition reactions gave (Z)- $\beta, \gamma$-unsaturated esters. ${ }^{10 \mathrm{e}}$ Thus, generally 1,6 -conjugate addition to 2,4 -pentadienoate has been believed to give the $(E)-\beta, \gamma$-unsaturated esters at least in the absence of transition-metal catalyst.

First, the stereochemistry of the nucleophilic addition of $\mathrm{Et}_{2} \mathrm{NH}$ to $\mathbf{4}$ was investigated in various solvents as listed in Table 4. Although the reactions were rather sluggish compared with the reaction of (E)-1-tosyl-1,3-butadiene (1), similar high $\mathrm{Z}$ selectivity was realized in polar and less bulky ethers, such
Table 5. Stereochemistry of Nucleophilic Addition of Various Amines 2 to $\mathbf{4}^{\text {a) }}$

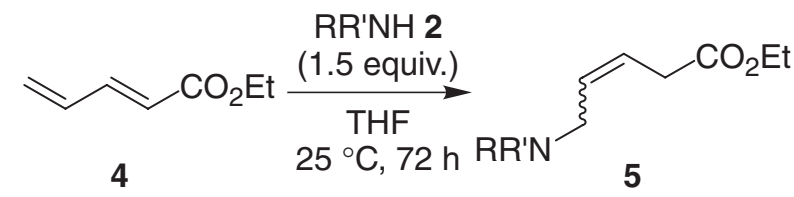

\begin{tabular}{cccccc}
\hline Entry & $\mathrm{RR}^{\prime} \mathrm{NH} \mathbf{2}$ & & $\mathbf{4}^{\text {b) }}$ & ${\text { Yield } / \%^{\mathrm{c})}}^{\mathrm{Z}} / \mathrm{E}^{\mathrm{d})}$ \\
\hline $1^{\mathrm{e})}$ & $n-\mathrm{PrNH}_{2}$ & $\mathbf{a}$ & $86 / 14$ & 10 & $7 / 93$ \\
$2^{\mathrm{e})}$ & $n-\mathrm{BuNH}_{2}$ & $\mathbf{b}$ & $89 / 11$ & 8 & $11 / 89$ \\
3 & $\mathrm{Me}_{2} \mathrm{NH}^{\mathrm{f})}$ & $\mathbf{c}$ & $52 / 48$ & 32 & $76 / 24$ \\
4 & $\mathrm{Et}_{2} \mathrm{NH}$ & $\mathbf{d}$ & $89 / 11$ & 9 & $87 / 13$ \\
5 & $n-\mathrm{Pr}_{2} \mathrm{NH}$ & $\mathbf{e}$ & $96 / 4$ & 4 & $90 / 10$ \\
6 & $n-\mathrm{Bu}_{2} \mathrm{NH}$ & $\mathbf{g}$ & $94 / 6$ & 5 & $94 / 6$ \\
7 & $n-\mathrm{Bu}(\mathrm{Me}) \mathrm{NH}$ & $\mathbf{h}$ & $66 / 34$ & 25 & $74 / 26$ \\
8 & $i-\mathrm{Pr}(\mathrm{Me}) \mathrm{NH}$ & $\mathbf{i}$ & $85 / 15$ & 15 & $82 / 18$ \\
9 & Pyrrolidine & $\mathbf{j}$ & $6 / 94$ & 90 & $59 / 41$ \\
10 & Piperidine & $\mathbf{k}$ & $39 / 61$ & 58 & $68 / 32$ \\
\hline
\end{tabular}

a) Concentration of amines 2 was $150 \mathrm{mM}$ in all cases. b) The ratios were determined based on the isolated yields. c) Isolated total yield of 5 based on $\mathbf{4}$. d) The ratios were determined by $400 \mathrm{MHz}{ }^{1} \mathrm{H}$ NMR spectra. ${ }^{11}$ e) Formation of 1-alkyl-1,6-dihydropyridin-2(3H)-one (alkyl $=n$-Pr, $5 \% ; n$-Bu, 6\%) was observed. f) A commercially available $2.0 \mathrm{M}$ solution of $\mathrm{Me}_{2} \mathrm{NH}$ in THF was used.

as THF and DME, to give the corresponding (Z)-5-amino-3pentenoate 5d selectively (Table 4, Entries 1 and 2).

In order to promote the addition reaction, several kinds of proton sources were added to activate the ester carbonyl group and/or to protonate to the anion developing at the $\alpha$-position. The reaction rate was remarkably accelerated by using proton sources with lower $\mathrm{p} K_{\mathrm{a}}$, however, $\mathrm{Z}$ selectivity of $\mathbf{5 d}$ was decreased in contrast. ${ }^{12}$

Although the addition reaction of $\mathrm{Et}_{2} \mathrm{NH}(\mathbf{2 d})$ proceeded with higher stereoselectivity, the chemical yield was poor. Next, we investigated the nucleophilic addition of various amines 2 to 4 in THF at $25^{\circ} \mathrm{C}$. The results are summarized in Table 5. Similar tendency toward $\mathrm{Z}$ selectivity in the case of (E)-1-tosyl-1,3-butadiene (1) was observed: Acyclic secondary amines, especially $n-\mathrm{Pr}_{2} \mathrm{NH}(\mathbf{2 e})$ and $n$ - $\mathrm{Bu}_{2} \mathrm{NH}(\mathbf{2 g})$, showed relatively high $\mathrm{Z}$ selectivity although the chemical yields were poorer (Entries 5 and 6). Pyrrolidine (2j), a cyclic secondary amine, showed high chemical yield, but $\mathrm{Z}$ selectivity was not particularly good (Entry 9). The reaction rate of nucleophilic addition of piperidine (2k) was moderate to afford relatively good $\mathrm{Z}$ selectivity (Entry 10 ).

Thus, the reaction of piperidine (2k) to 4 was examined in detail paying attention to the effect of temperature and concentration, and the results are summarized in Table 6 . It is noteworthy that the $\mathrm{Z}$ selectivity of $\mathbf{5 k}$ was again enhanced when the reaction was carried out at higher temperature (Entries $2-4,7$ and 8 ) and at lower concentration of piperidine (Entries 1 and 3, 5 and 6) up to $89 / 11$ in $50 \%$ yield. On the other hand, the concentration of 4 little affected the $\mathrm{Z} / \mathrm{E}$ ratio of 5k (Entries 3 and 5, 6 and 7, and 8 and 9, respectively).

As described above, nucleophilic addition of secondary amines to electron-deficient dienes $\mathbf{1}$ and $\mathbf{4}$ realized unprecedented high $\mathrm{Z}$ selectivity to give the corresponding sterically 
Table 6. Effect of Temperature and Concentration on Nucleophilic Addition of Piperidine (2k) to 4

\begin{tabular}{|c|c|c|c|c|c|c|c|}
\hline \multirow{2}{*}{ Entry } & \multicolumn{2}{|c|}{ Concentrations } & \multirow{2}{*}{$2 k / 4$} & \multirow{2}{*}{$\mathrm{Temp} /{ }^{\circ} \mathrm{C}$} & \multirow{2}{*}{$4 / 5 k^{a)}$} & \multirow{2}{*}{ Yield $/ \%^{\mathrm{b})}$} & \multirow{2}{*}{$\mathrm{Z} / \mathrm{E}^{\mathrm{c})}$} \\
\hline & $4 / \mathrm{mM}$ & $\mathbf{2 k} / \mathrm{mM}$ & & & & & \\
\hline 1 & 100 & 600 & 6.0 & 25 & $0 / 100$ & 99 & $34 / 66$ \\
\hline 2 & 100 & 150 & 1.5 & 0 & $47 / 53$ & 38 & $29 / 71$ \\
\hline 3 & 100 & 150 & 1.5 & 25 & $39 / 61$ & 58 & $68 / 32$ \\
\hline 4 & 100 & 150 & 1.5 & 60 & $14 / 86$ & 74 & $72 / 28$ \\
\hline 5 & 25 & 150 & 6.0 & 25 & $18 / 82$ & 67 & $65 / 35$ \\
\hline 6 & 25 & 37.5 & 1.5 & 25 & $72 / 28$ & 21 & $83 / 17$ \\
\hline 7 & 6.25 & 37.5 & 6.0 & 25 & $72 / 28$ & 25 & $81 / 19$ \\
\hline 8 & 6.25 & 37.5 & 6.0 & 60 & $44 / 56$ & 50 & $89 / 11$ \\
\hline 9 & 3.125 & 37.5 & 12.0 & 60 & $35 / 65$ & 55 & $87 / 13$ \\
\hline
\end{tabular}

a) The ratios were determined based on the isolated yields. b) Isolated total yield of $\mathbf{5 k}$ based on $\mathbf{4}$. c) The ratios were determined by $400 \mathrm{MHz}{ }^{1} \mathrm{HNMR}$ spectra.

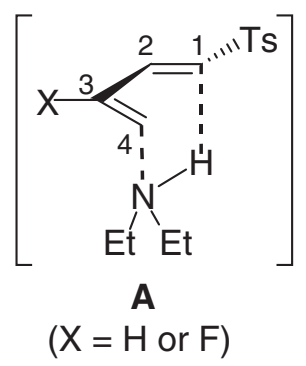

Figure 2. Another possible origin of the "syn effect:" 1,4addition.

unfavorable ( $Z$ )-allylic compounds $\mathbf{3 d}$ and $\mathbf{5 k}$. The mechanism for predominant formation of $(Z)$-olefins is not yet clear. ${ }^{13}$ To confirm the possibility of a concerted 1,4-addition mechanism (Figure 2, A), nucleophilic addition of $\mathrm{Et}_{2} \mathrm{NH}(\mathbf{2 d})(150 \mathrm{mM})$ to (E)-3-fluoro-1-tosyl-1,3-butadiene (6) was investigated (eq 1). Selective formation of (Z)-3-fluoro-2-butenyl sulfone derivative ( $Z$ )-7 was observed. In addition, nucleophilic addition of piperidine $(\mathbf{2 k})(150 \mathrm{mM})$ to ethyl $(E)$-4-fluoro-2,4pentadienoate $(\mathbf{8})(100 \mathrm{mM})$ also gave the corresponding $\mathrm{Z}$ adduct, (Z)-9, selectively (eq 2 ). Selective formations of $(Z$ )olefins, $(Z)-7$ and (Z)-9, could exclude the 1,4-addition mechanism A.

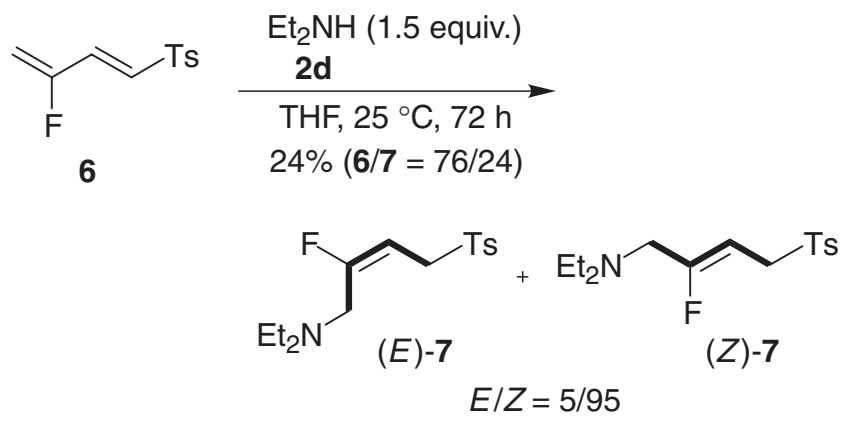<smiles>C=C(F)C=CC(=O)OCC</smiles><smiles>CCOC(=O)CC=C(F)CN1CCC[C@H](CC=C(F)CN2CCCCC2)C1</smiles>

The elucidation of selective formation of $(Z)$-allylic sulfone (Z)-3d and (Z)-3-pentenoate ( $Z$ )-5k and variation of $Z / E$ ratios by kinds of amines, solvents, temperature, and concentration are quite difficult. The most favorable conformation of $(E)$-dienyl sulfone 1 and (E)-2,4-pentadienoate 4 might be s-trans conformation. ${ }^{14}$ If the present addition reaction were kinetically controlled reflecting the initial conformation shown in $\mathbf{C}$ (Scheme 1), it would be impossible to explain the $\mathrm{Z}$ selectivity. We try to rationalize the origin of the present "syn effect" in two ways, $\mathrm{n} / \sigma \rightarrow \pi^{*}$ interaction and/or $6 \pi$-electron homoaromaticity in the following, ${ }^{15}$ taking into account the effects of kinds of amines, solvents, temperature, and concentration.

The former is as follows (Scheme 1): When a pair of electrons on the amine nitrogen interacts with a $\pi^{*}$ orbital of $\mathrm{C}_{\gamma}=\mathrm{C}_{\delta}$ at the $\delta$-position of $\mathbf{1}$ and $\mathbf{4}$ via s-trans conformation as shown in $\mathbf{C}$, an anion would develop on the $\gamma$-carbon which might acquire $\mathrm{sp}^{3}$ character altering from $\mathrm{sp}^{2}$ character. The nonbonding electron pair (n) of the $\gamma$-carbanion can more effectively interact with the $\pi^{*}$ orbital of $\mathrm{C}_{\alpha}=\mathrm{C}_{\beta}$ in the eclipsed conformations $\mathbf{D}$ and $\mathbf{F}$, in both of which the n-orbital is aligned with the $\pi^{*}$ orbital ( $\mathrm{n} \rightarrow \pi^{*}$ interaction), and conformation $\mathbf{E}$ can be neglected. ${ }^{16}$ Further, the contribution of $\sigma \rightarrow$ $\pi^{*}$ interaction might determine the preference of $\mathbf{D}$ or $\mathbf{F}$, 


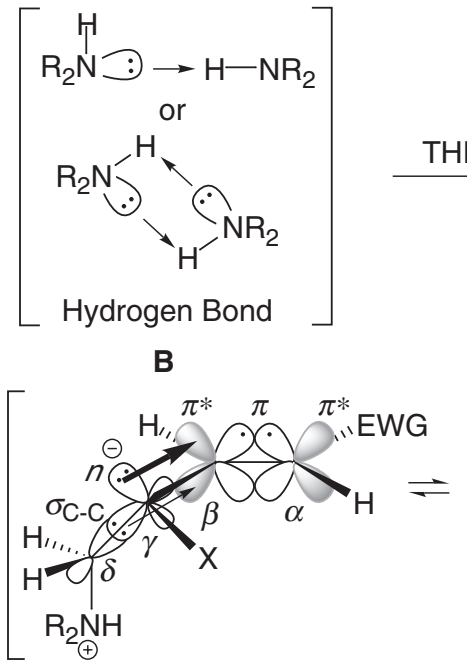

D

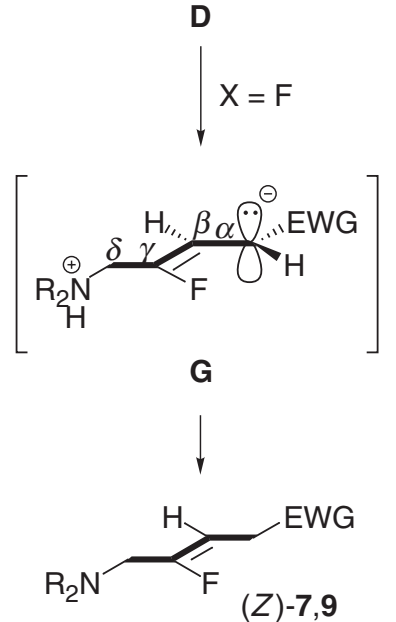

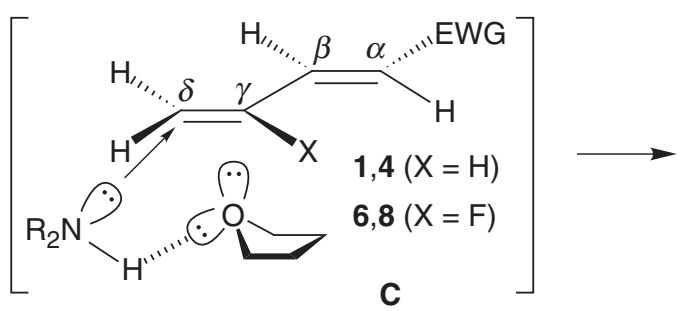

C

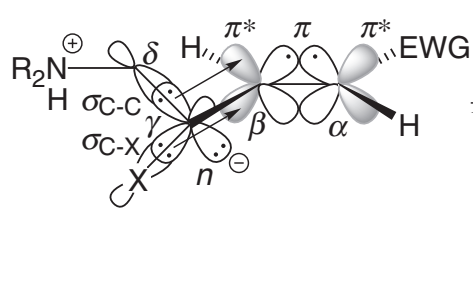

E

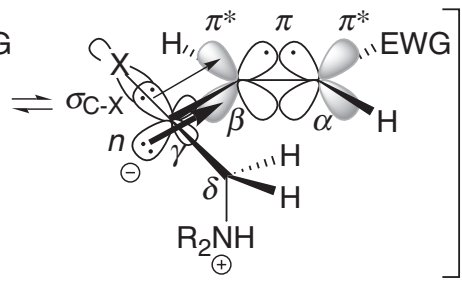

$\mathbf{F}$

$\sigma_{\mathrm{C}-\mathrm{X}} \rightarrow \pi^{\star}$ interaction $\mathrm{X}=\mathrm{H}>\mathrm{C}>\mathrm{F}$

$\mathrm{EWG}=\mathrm{Ts}$ or $\mathrm{CO}_{2} \mathrm{Et}$
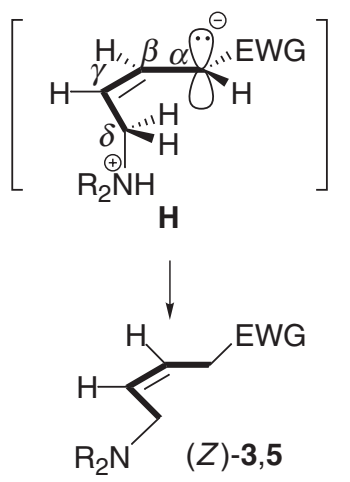

Scheme 1.

because $\sigma \rightarrow \pi^{*}$ interactions decrease in the order of $\sigma_{\mathrm{C}-\mathrm{H}} \rightarrow \pi^{*}>\sigma_{\mathrm{C}-\mathrm{C}} \rightarrow \pi^{*}>\sigma_{\mathrm{C}-\mathrm{F}} \rightarrow \pi^{*} .{ }^{17}$ Thus, $(Z)-3$ and $(Z)-5$ were predominantly obtained in the case of $\mathbf{1}$ and $\mathbf{4}$ via conformation $\mathbf{F}(\mathrm{X}=\mathrm{H})$, whereas $(Z)-\mathbf{7}$ and $(Z)-\mathbf{9}$ were formed in the case of $\mathbf{6}$ and $\mathbf{8}$ via $\mathbf{D}(X=F)$. Higher temperature and lower concentration in coordinating solvent like THF might dissociate the aggregation of bulkier dialkylamine (B) via hydrogen bonding ${ }^{18}$ to generate the more nucleophilic monomeric amine shown in $\mathbf{C}{ }^{19}$

In addition, $6 \pi$-electron homoaromaticity seems to also contribute to the "syn effect" (Figure 3). When a monomeric dialkylamine reacts with an electron deficient diene, the syn intermediate $\mathbf{J}$ might be stabilized by $6 \pi$-electron homoaromaticity, followed by protonation to afford the corresponding $(Z)$ 3 and $(Z)-5$. In the case of $\gamma$-fluorinated dienes $\mathbf{6}$ and $\mathbf{8}$, the participation of a lone pair of electrons on $\gamma$-fluorine atom to $6 \pi$-electron homoaromaticity, as depicted in $\mathbf{L}$, may be much more effective than that of methylene in $\mathbf{K}$. As a result, $(Z)-7$ and (Z)-9 were selectively obtained.

Furthermore, the reaction of $\mathrm{Et}_{2} \mathrm{NH}(\mathbf{2 d})(150 \mathrm{mM})$ with (E)-1-fluoro-1-tosyl-1,3-butadiene (10) was examined, and (Z)-allylic sulfone $\mathbf{1 1}$ was mainly obtained (eq 3). Addition of piperidine $(150 \mathrm{mM})$ to octyl $(Z)$-2-fluoro-2,4-pentadienoate (12) mainly gave (E)-2-fluoro-5-amino-3-pentenoate 13
(Table 7, Entry 1), whereas Z selectivity was enhanced under dilute conditions at higher temperature as shown in Table $7 .^{20}$ Their syn intermediate forms $8 \pi$-electron system $\mathbf{N}$, which has no advantage compared with anti intermediate $\mathbf{M}$ leading to $(E)$-allylic products. The fact that a considerable amount of $\mathrm{Z}$ isomers were still produced might suggest the contribution of $n / \sigma \rightarrow \pi^{*}$ interaction discussed above, or that of a hydrogen bond between the $\alpha$-fluorine atom and a proton of ammonium ion as depicted in $\mathbf{O}$.

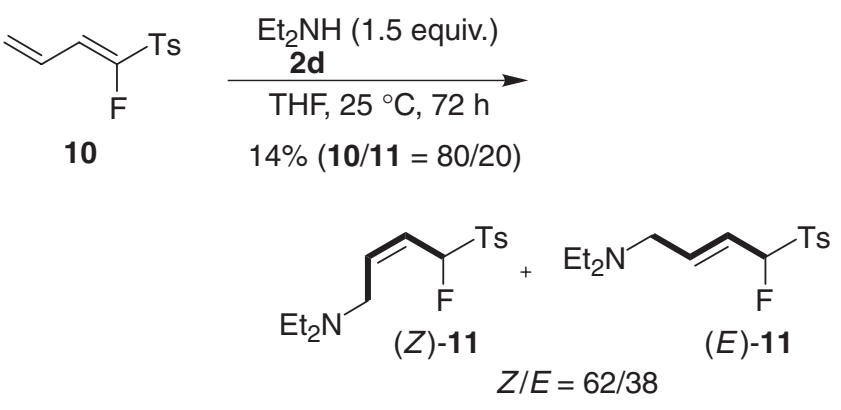

In conclusion, unprecedented $\mathrm{Z}$ selective conjugate addition of amines to (E)-1-tosyl-1,3-butadiene (1) and ethyl (E)-2,4pentadienoate (4) was discovered. Especially, higher Z selec- 

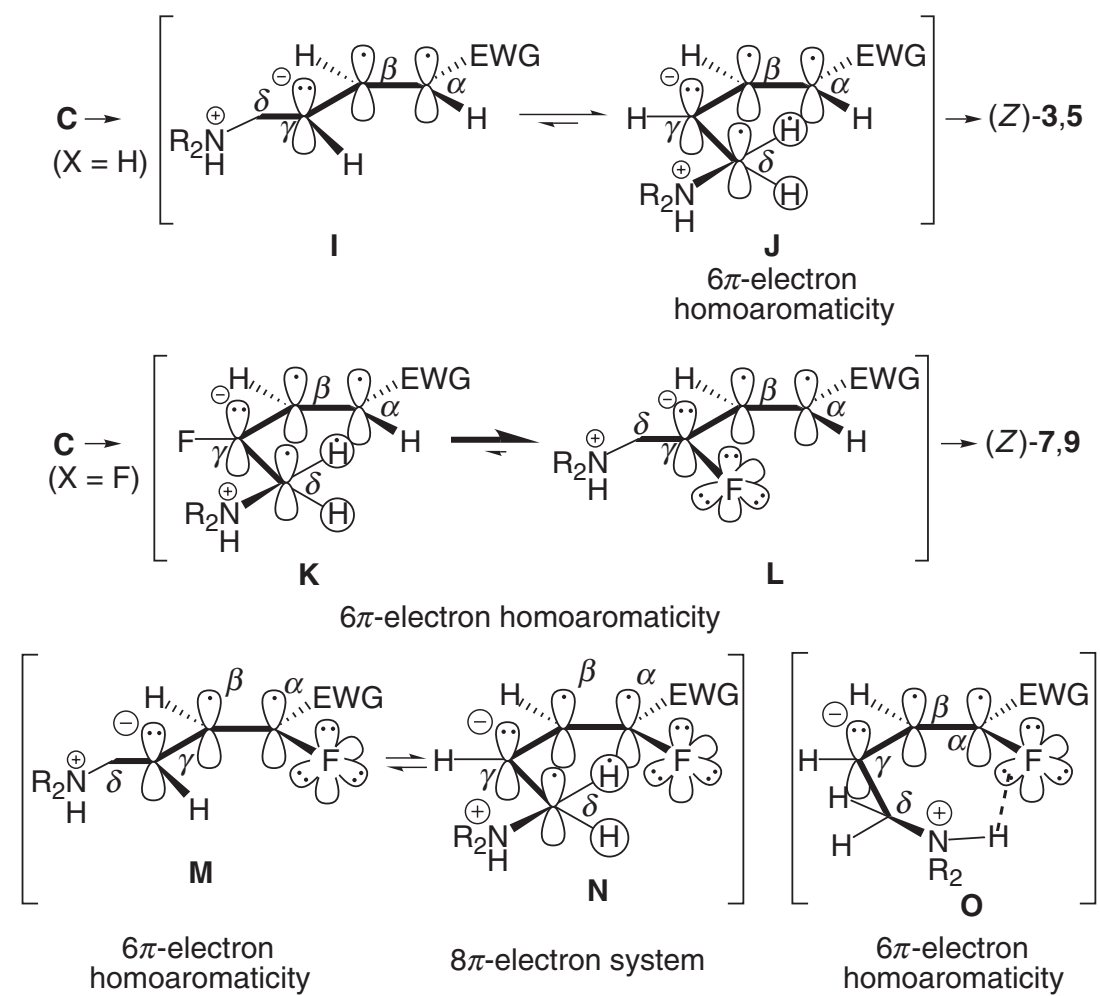

Figure 3. "Syn effect" based on $6 \pi$-electron homoaromaticity.

Table 7. Effect of Temperature and Concentration on Nucleophilic Addition of Piperidine (2k) to 12

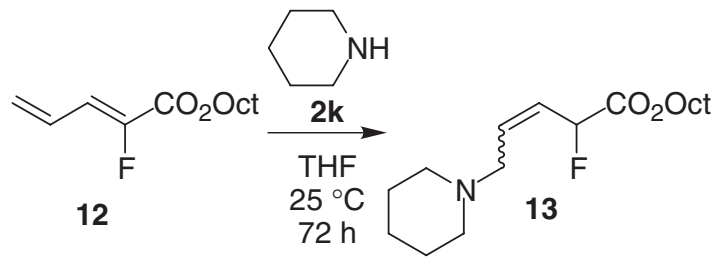

\begin{tabular}{|c|c|c|c|c|c|c|c|}
\hline \multirow{2}{*}{ Entry } & \multicolumn{2}{|c|}{ Concentrations } & \multirow{2}{*}{$2 k / 12$} & \multirow{2}{*}{ Temp $/{ }^{\circ} \mathrm{C}$} & \multirow{2}{*}{$12 / 13^{a)}$} & \multirow{2}{*}{ Yield $/ \%^{\text {b) }}$} & \multirow{2}{*}{$\mathrm{Z} / \mathrm{E}^{\mathrm{c})}$} \\
\hline & $12 / \mathrm{mM}$ & $\mathbf{2 k} / \mathrm{mM}$ & & & & & \\
\hline 1 & 100 & 150 & 1.5 & 25 & $66 / 34$ & 33 & $18 / 82$ \\
\hline 2 & 100 & 150 & 1.5 & 60 & $26 / 74$ & 73 & $20 / 80$ \\
\hline 3 & 25 & 37.5 & 1.5 & 25 & $97 / 3$ & 3 & $32 / 68$ \\
\hline 4 & 6.25 & 37.5 & 6.0 & 25 & $96 / 4$ & 4 & $35 / 65$ \\
\hline 5 & 6.25 & 37.5 & 6.0 & 60 & $88 / 12$ & 12 & $50 / 50$ \\
\hline
\end{tabular}

a) The ratios were determined based on the isolated yields. b) Isolated total yield of $\mathbf{1 3}$ based on 12. c) The ratios were determined by $400 \mathrm{MHz}{ }^{1} \mathrm{H} \mathrm{NMR}$ spectra.

tivity could be realized when the reaction was carried out in lower concentration of secondary amines at higher temperature in polar ethereal solvent. The "syn effect" observed in the present addition reaction was rationalized by $\mathrm{n} / \sigma \rightarrow \pi^{*}$ interaction and/or $6 \pi$-electron homoaromaticity.

\section{Experimental}

${ }^{1} \mathrm{H}$ and ${ }^{19} \mathrm{~F}$ NMR spectra were recorded on a JEOL Lambda $400 \mathrm{NMR}$ spectrometer $\left(400 \mathrm{MHz}\right.$ for ${ }^{1} \mathrm{H}$ and $376 \mathrm{MHz}$ for $\left.{ }^{19} \mathrm{~F}\right)$. Chemical shifts were determined in the $\delta$-scale relative to $\mathrm{Si}\left(\mathrm{CH}_{3}\right)_{4}(\delta 0)$ and $\mathrm{C}_{6} F_{6}(\delta-162.90)$ as internal standards, respectively. IR spectra were measured on a JASCO FT/IR-230 spectrometer. MS spectra were recorded with a JEOL SX-102A mass spectrometer. THF and $\mathrm{Et}_{2} \mathrm{O}$ were freshly distilled from sodium diphenylketyl. All other solvents were distilled and stored over drying agents. Thin-layer chromatography (TLC), flash column chromatography, and recycle HPLC were performed by using Merck silica gel $60 \mathrm{PF}_{254}$ (Art. 7749), Cica silica gel 60 (No. 37571), and JAIGL-SIL (s-043-15), respectively.

$(E)$-1-Tosyl-1,3-butadiene (1). ${ }^{21}$ To a solution of $(E)$-1-iodo4-tosyl-2-butene ${ }^{5 a}(758 \mathrm{mg}, 2.25 \mathrm{mmol})$ in THF $(7.0 \mathrm{~mL})$ was added 1,8-diazabicyclo[5.4.0]undec-7-ene (DBU) $\quad(0.370 \mathrm{~mL}$, $2.48 \mathrm{mmol}$ ) under a nitrogen atmosphere. After $5 \mathrm{~min}$, the reaction mixture was treated with a saturated aqueous solution of $\mathrm{NH}_{4} \mathrm{Cl}$, and the solvent was evaporated. The organic substances were extracted with EtOAc, followed by washing with $\mathrm{H}_{2} \mathrm{O}$, brine, and dried over $\mathrm{Na}_{2} \mathrm{SO}_{4}$. After evaporating the solvent, the residue 
was purified by column chromatography $\left(\mathrm{SiO}_{2}\right.$, hexane/ EtOAc $=5 / 1, v / v)$ to give $\mathbf{1}$ in $99 \%$ yield $(465 \mathrm{mg})$ as an oil. IR (neat) 3050, 2923, 2850, 1596, 1493, 1449, 1425, 1410, 1303, 1182, 1147, 1087, 1020, 969, 890, 821, 728, $669 \mathrm{~cm}^{-1}$. ${ }^{1} \mathrm{HNMR}\left(\mathrm{CDCl}_{3}\right) \delta 2.44(3 \mathrm{H}, \mathrm{s}), 5.59(1 \mathrm{H}, \mathrm{d}, J=10.25 \mathrm{~Hz})$, $5.71(1 \mathrm{H}, \mathrm{d}, J=16.83 \mathrm{~Hz}), 6.37(1 \mathrm{H}, \mathrm{ddd}, J=16.83,11.22$, $10.25 \mathrm{~Hz}), 6.38(1 \mathrm{H}, \mathrm{d}, J=14.88 \mathrm{~Hz}), 7.23(1 \mathrm{H}, \mathrm{dd}, J=14.88$, $11.22 \mathrm{~Hz}), 7.34(2 \mathrm{H}, \mathrm{d}, J=8.24 \mathrm{~Hz}), 7.78(2 \mathrm{H}, \mathrm{d}, J=8.24 \mathrm{~Hz})$. HRMS $\left(\mathrm{FAB}^{+}\right)(\mathrm{M}+\mathrm{H})^{+}$, Found: $m / z$ 209.06386. Calcd for $\mathrm{C}_{11} \mathrm{H}_{13} \mathrm{O}_{2} \mathrm{~S}$ : 209.06363 .

Representative Procedure for the Nucleophilic Addition Reaction of Diethylamine (2d) to $(E)$-1-Tosyl-1,3-butadiene (1) (Table 1, Entry 4). To a solution of (E)-1-tosyl-1,3-butadiene (1) $(94 \mathrm{mg}, 0.45 \mathrm{mmol})$ in THF $(4.5 \mathrm{~mL})$ was added diethylamine (2d) $(0.070 \mathrm{~mL}, 0.68 \mathrm{mmol})$ at $25^{\circ} \mathrm{C}$ under a nitrogen atmosphere. After stirring for $72 \mathrm{~h}$, the reaction mixture was quenched by adding silica gel $(3.00 \mathrm{~g})$, and the solvent was evaporated. The residue was purified by column chromatography $\left(\mathrm{SiO}_{2}\right.$, hexane/ EtOAc $=5 / 1, \mathrm{v} / \mathrm{v}$, EtOAc, then EtOAc $/ \mathrm{MeOH}=3 / 1, \mathrm{v} / \mathrm{v}$ ) to give a mixture of $(Z)$ - and $(E)-3 d(95 \mathrm{mg}, 75 \%, \mathrm{Z} / \mathrm{E}=74 / 26)$ and to recover unreacted $\mathbf{1}(17 \mathrm{mg}, 18 \%)$.

In a similar way, addition reaction of amines $\mathbf{2 a}-\mathbf{2} \mathbf{c}$ and $\mathbf{2 e}-\mathbf{2} \mathbf{k}$ was carried out to give the corresponding allylic amines $\mathbf{3 a}-\mathbf{3 c}$ and $\mathbf{3 e}-\mathbf{3 k}$. The physical and spectral data of $\mathbf{3 a}-\mathbf{3 e}$ and $\mathbf{3 g}-\mathbf{3 k}$ and by-products are given in the following. $\mathrm{Z} / \mathrm{E}$ ratios were determined by $400 \mathrm{MHz}{ }^{1} \mathrm{H}$ NMR spectra.

$N$-Propyl-4-tosyl-2-butenylamine (3a): An oil (Z/E $=21$ / 79). IR (neat) 3321, 2958, 2929, 2874, 2810, 1597, 1495, 1455, $1403,1381,1317,1303,1238,1145,1088,1018,973,816$, $730,665 \mathrm{~cm}^{-1} . \mathrm{Z}$ isomer; ${ }^{1} \mathrm{HNMR}\left(\mathrm{CDCl}_{3}\right) \delta 0.87(3 \mathrm{H}, \mathrm{t}$, $J=7.32 \mathrm{~Hz}), 1.41(2 \mathrm{H}$, sext, $J=7.32 \mathrm{~Hz}), 1.56$ (1H, brs), 2.40 $(2 \mathrm{H}, \mathrm{t}, J=7.32 \mathrm{~Hz}), 2.44(3 \mathrm{H}, \mathrm{s}), 3.00(2 \mathrm{H}, \mathrm{dd}, J=6.83$, $1.46 \mathrm{~Hz}), 3.90(2 \mathrm{H}, \mathrm{d}, J=8.05 \mathrm{~Hz}), 5.49(1 \mathrm{H}, \mathrm{dtt}, J=10.73$, $8.05,1.46 \mathrm{~Hz}), 5.88(1 \mathrm{H}, \mathrm{dt}, J=10.73,6.83 \mathrm{~Hz}), 7.34(2 \mathrm{H}, \mathrm{d}$, $J=7.81 \mathrm{~Hz}), 7.75(2 \mathrm{H}, \mathrm{d}, J=7.81 \mathrm{~Hz}) . \mathrm{E}$ isomer; ${ }^{1} \mathrm{HNMR}$ $\left(\mathrm{CDCl}_{3}\right) \delta 0.89(3 \mathrm{H}, \mathrm{t}, J=7.32 \mathrm{~Hz}), 1.45(2 \mathrm{H}, \quad$ sext, $J=$ $7.32 \mathrm{~Hz}), 1.56(1 \mathrm{H}, \mathrm{brs}), 2.44(3 \mathrm{H}, \mathrm{s}), 2.46(2 \mathrm{H}, \mathrm{t}, J=7.32 \mathrm{~Hz})$, $3.19(2 \mathrm{H}, \mathrm{d}, J=4.64 \mathrm{~Hz}), 3.77(2 \mathrm{H}, \mathrm{d}, J=6.10 \mathrm{~Hz}), 5.58(1 \mathrm{H}$, $\mathrm{dt}, J=15.37,6.10 \mathrm{~Hz}), 5.63(1 \mathrm{H}, \mathrm{dt}, J=15.37,4.64 \mathrm{~Hz}), 7.33$ $(2 \mathrm{H}, \mathrm{d}, J=7.81 \mathrm{~Hz}), 7.73(2 \mathrm{H}, \mathrm{d}, J=7.81 \mathrm{~Hz}) . \mathrm{HRMS}\left(\mathrm{FAB}^{+}\right)$ $(\mathrm{M}+\mathrm{H})^{+}$, Found: $m / z$ 268.13820. Calcd for $\mathrm{C}_{14} \mathrm{H}_{22} \mathrm{NO}_{2} \mathrm{~S}$ : 268.13712.

$\boldsymbol{N}$-Propyl-4-tosyl- $\boldsymbol{N}$-(4-tosyl-2-butenyl)-2-butenylamine: An oil (a mixture of at least 3 isomers based on ${ }^{1} \mathrm{H}$ NMR). IR (neat) 2960, 2929, 2872, 1597, 1495, 1455, 1403, 1316, 1235, 1142, 1087, 1018, 976, 881, 815, 728, 666 $\mathrm{cm}^{-1} .{ }^{1} \mathrm{HNMR}\left(\mathrm{CDCl}_{3}\right)$ $\delta 0.76-0.81(2 \mathrm{H}, \mathrm{m}), 0.79(3 \mathrm{H}, \mathrm{t}, J=7.32 \mathrm{~Hz}),[0.80(3 \mathrm{H}, \mathrm{t}$, $J=7.32 \mathrm{~Hz})$, minor isomers], 2.09-2.19 $(2 \mathrm{H}, \mathrm{m}), 2.44(6 \mathrm{H}, \mathrm{s})$, [2.46 $(6 \mathrm{H}, \mathrm{s})$, minor isomers], $2.86(2 \mathrm{H}, \mathrm{d}, J=3.90 \mathrm{~Hz}), 2.91$ $(2 \mathrm{H}, \mathrm{d}, J=3.42 \mathrm{~Hz}),[2.69-2.76(4 \mathrm{H}, \mathrm{m})$, minor isomers], 3.74$3.78(4 \mathrm{H}, \mathrm{m}),[3.83-3.87(4 \mathrm{H}, \mathrm{m})$, minor isomers], 5.48-5.79 $(4 \mathrm{H}, \mathrm{m}), 7.32-7.36(4 \mathrm{H}, \mathrm{m}), 7.70-7.68(4 \mathrm{H}, \mathrm{m}) . \mathrm{HRMS}\left(\mathrm{FAB}^{+}\right)$ $(\mathrm{M}+\mathrm{H})^{+}$, Found: $m / z$ 476.19165. Calcd for $\mathrm{C}_{25} \mathrm{H}_{34} \mathrm{NO}_{4} \mathrm{~S}_{2}$ : 476.19293.

N-Butyl-4-tosyl-2-butenylamine (3b): An oil $(\mathrm{Z} / \mathrm{E}=23$ / 77). IR (neat) 3323, 3030, 2956, 2927, 2871, 2810, 1597, 1493, 1457, 1402, 1377, 1318, 1303, 1240, 1146, 1088, 1045, 1018, $972,917,875,816,731,695,664 \mathrm{~cm}^{-1} . \mathrm{Z}$ isomer; ${ }^{1} \mathrm{HNMR}$ $\left(\mathrm{CDCl}_{3}\right) \delta 0.89(3 \mathrm{H}, \mathrm{t}, J=7.07 \mathrm{~Hz}), 1.31(2 \mathrm{H}, \quad$ sext, $J=$ $7.07 \mathrm{~Hz}), 1.43(2 \mathrm{H}$, quint, $J=7.07 \mathrm{~Hz}), 1.73(1 \mathrm{H}$, brs $), 2.44$ $(3 \mathrm{H}, \mathrm{s}), 2.51(2 \mathrm{H}, \mathrm{t}, J=7.07 \mathrm{~Hz}), 3.01(2 \mathrm{H}, \mathrm{dd}, J=6.83$, $1.46 \mathrm{~Hz}), 3.89(2 \mathrm{H}, \mathrm{d}, J=7.81 \mathrm{~Hz}), 5.49(1 \mathrm{H}, \mathrm{dtt}, J=10.98$,
$7.81,1.46 \mathrm{~Hz}), 5.88(1 \mathrm{H}, \mathrm{dt}, J=10.98,6.83 \mathrm{~Hz}), 7.34(2 \mathrm{H}, \mathrm{d}$, $J=7.81 \mathrm{~Hz}), 7.75(2 \mathrm{H}, \mathrm{d}, J=7.81 \mathrm{~Hz})$. E isomer; ${ }^{1} \mathrm{HNMR}$ $\left(\mathrm{CDCl}_{3}\right) \delta 0.91(3 \mathrm{H}, \mathrm{t}, J=7.07 \mathrm{~Hz}), 1.31(2 \mathrm{H}$, sext, $J=$ $7.07 \mathrm{~Hz}), 1.43(2 \mathrm{H}$, quint, $J=7.07 \mathrm{~Hz}), 1.73(1 \mathrm{H}$, brs $), 2.44$ $(3 \mathrm{H}, \mathrm{s}), 2.51(2 \mathrm{H}, \mathrm{t}, J=7.07 \mathrm{~Hz}), 3.21(2 \mathrm{H}, \mathrm{d}, J=4.64 \mathrm{~Hz})$, $3.77(2 \mathrm{H}, \mathrm{d}, J=6.34 \mathrm{~Hz}), 5.59(1 \mathrm{H}, \mathrm{dt}, J=15.61,6.34 \mathrm{~Hz})$, $5.64(1 \mathrm{H}, \mathrm{dt}, J=15.61,4.64 \mathrm{~Hz}), 7.34(2 \mathrm{H}, \mathrm{d}, J=7.81 \mathrm{~Hz})$, $7.74(2 \mathrm{H}, \mathrm{d}, J=7.81 \mathrm{~Hz})$. HRMS $\left(\mathrm{FAB}^{+}\right)(\mathrm{M}+\mathrm{H})^{+}$, Found: $m / z$ 282.15262. Calcd for $\mathrm{C}_{15} \mathrm{H}_{24} \mathrm{NO}_{2} \mathrm{~S}: 282.15277$.

$\mathrm{N}$-Butyl-4-tosyl- $\boldsymbol{N}$-(4-tosyl-2-butenyl)-2-butenylamine: An oil (a mixture of at least 3 isomers based on ${ }^{1} \mathrm{HNMR}$ ). IR (neat) 3030, 2955, 2927, 2871, 2813, 1597, 1494, 1455, 1403, 1316, 1303, 1237, 1142, 1087, 1018, 975, 882, 816, 728, 690, $664 \mathrm{~cm}^{-1} .{ }^{1} \mathrm{HNMR}\left(\mathrm{CDCl}_{3}\right) \delta 0.85(3 \mathrm{H}, \mathrm{t}, J=7.08 \mathrm{~Hz}),[0.86$ $(3 \mathrm{H}, \mathrm{t}, J=7.08 \mathrm{~Hz})$, minor isomers], 1.14-1.34 (4H, m), 2.15$2.23(2 \mathrm{H}, \mathrm{m}), 2.44(6 \mathrm{H}, \mathrm{s}),[2.46(6 \mathrm{H}, \mathrm{s})$, minor isomers], 2.86 $(2 \mathrm{H}, \mathrm{d}, J=3.39 \mathrm{~Hz}), 2.91(2 \mathrm{H}, \mathrm{d}, J=3.17 \mathrm{~Hz}),[2.69-2.76$ $(4 \mathrm{H}, \mathrm{m})$, minor isomers], 3.72-3.79 $(4 \mathrm{H}, \mathrm{m}),[3.82-3.88(4 \mathrm{H}$, $\mathrm{m})$, minor isomers], 5.45-5.79 $(4 \mathrm{H}, \mathrm{m}), 7.32-7.36(4 \mathrm{H}, \mathrm{m})$, 7.70-7.76 (4H, m). HRMS $\left(\mathrm{FAB}^{+}\right)(\mathrm{M}+\mathrm{H})^{+}$, Found: $m / z$ 490.20833. Calcd for $\mathrm{C}_{26} \mathrm{H}_{36} \mathrm{NO}_{4} \mathrm{~S}_{2}: 490.20858$.

$N, N$-Dimethyl-4-tosyl-2-butenylamine (3c): An oil (Z/ $\mathrm{E}=60 / 40$ ). IR (neat) 3031, 2943, 2860, 2818, 2773, 1597, 1494, 1458, 1402, 1317, 1239, 1148, 1088, 1019, 977, 891, 852, 816, 713, 689, $663 \mathrm{~cm}^{-1} . \mathrm{Z}$ isomer; ${ }^{1} \mathrm{HNMR}\left(\mathrm{CDCl}_{3}\right) \delta 2.07$ $(6 \mathrm{H}, \mathrm{s}), 2.44(3 \mathrm{H}, \mathrm{s}), 2.67(2 \mathrm{H}, \mathrm{dd}, J=6.58,1.46 \mathrm{~Hz}), 3.90$ $(2 \mathrm{H}, \mathrm{d}, J=8.05 \mathrm{~Hz}), 5.54(1 \mathrm{H}, \mathrm{dtt}, J=10.98,8.05,1.46 \mathrm{~Hz})$, $5.82(1 \mathrm{H}, \mathrm{dt}, J=10.98,6.58 \mathrm{~Hz}), 7.34(2 \mathrm{H}, \mathrm{d}, J=8.29 \mathrm{~Hz})$, $7.76(2 \mathrm{H}, \mathrm{d}, J=8.29 \mathrm{~Hz})$. E isomer; ${ }^{1} \mathrm{HNMR}\left(\mathrm{CDCl}_{3}\right) \delta 2.12$ $(6 \mathrm{H}, \mathrm{s}), 2.44(3 \mathrm{H}, \mathrm{s}), 2.87(2 \mathrm{H}, \mathrm{d}, J=3.90 \mathrm{~Hz}), 3.79(2 \mathrm{H}, \mathrm{d}$, $J=6.10 \mathrm{~Hz}), 5.52-5.60(1 \mathrm{H}, \mathrm{m}), 5.60(1 \mathrm{H}, \mathrm{dt}, J=15.34$, $6.10 \mathrm{~Hz}), 7.33(2 \mathrm{H}, \mathrm{d}, J=8.29 \mathrm{~Hz}), 7.73(2 \mathrm{H}, \mathrm{d}, J=8.29 \mathrm{~Hz})$. HRMS $\left(\mathrm{FAB}^{+}\right)(\mathrm{M}+\mathrm{H})^{+}$, Found: $m / z$ 254.12169. Calcd for $\mathrm{C}_{13} \mathrm{H}_{20} \mathrm{NO}_{2} \mathrm{~S}$ : 254.12147 .

$N, N$-Diethyl-4-tosyl-2-butenylamine (3d): $\quad$ An oil $(\mathrm{Z} / \mathrm{E}=$ 74/26). IR (neat) 3030, 2970, 2932, 2873, 2807, 1597, 1494, 1452, 1384, 1317, 1303, 1239, 1200, 1141, 1088, 1019, 976, $880,816,769,712,688 \mathrm{~cm}^{-1} . \mathrm{Z}$ isomer; ${ }^{1} \mathrm{HNMR}\left(\mathrm{CDCl}_{3}\right)$ $\delta 0.91(6 \mathrm{H}, \mathrm{t}, J=7.32 \mathrm{~Hz}), 2.31(4 \mathrm{H}, \mathrm{q}, J=7.32 \mathrm{~Hz}), 2.44$ $(3 \mathrm{H}, \mathrm{s}), 2.76(2 \mathrm{H}, \mathrm{d}, J=6.59 \mathrm{~Hz}), 3.89(2 \mathrm{H}, \mathrm{d}, J=7.81 \mathrm{~Hz})$, $5.53(1 \mathrm{H}, \mathrm{dt}, J=10.00,7.81 \mathrm{~Hz}), 5.83(1 \mathrm{H}, \mathrm{dt}, J=10.00$, $6.59 \mathrm{~Hz}), 7.34(2 \mathrm{H}, \mathrm{d}, J=8.05 \mathrm{~Hz}), 7.76(2 \mathrm{H}, \mathrm{d}, J=8.05 \mathrm{~Hz})$. ${ }^{1} \mathrm{HNMR}\left(\mathrm{C}_{6} \mathrm{D}_{6}\right) \delta 0.79(6 \mathrm{H}, \mathrm{t}, J=7.08 \mathrm{~Hz}), 1.86(3 \mathrm{H}, \mathrm{s}), 2.12$ $(4 \mathrm{H}, \mathrm{q}, J=7.08 \mathrm{~Hz}), 2.58(2 \mathrm{H}, \mathrm{d}, J=6.34 \mathrm{~Hz}), 3.64(2 \mathrm{H}, \mathrm{d}$, $J=7.81 \mathrm{~Hz}), 5.42(1 \mathrm{H}, \mathrm{dt}, J=10.98,7.81 \mathrm{~Hz}), 5.65(1 \mathrm{H}, \mathrm{dt}$, $J=10.98,6.34 \mathrm{~Hz}), 6.74(2 \mathrm{H}, \mathrm{d}, J=8.05 \mathrm{~Hz}), 7.70(2 \mathrm{H}, \mathrm{d}$, $J=8.05 \mathrm{~Hz})$. E isomer; ${ }^{1} \mathrm{HNMR}\left(\mathrm{CDCl}_{3}\right) \delta 0.95(6 \mathrm{H}, \mathrm{t}$, $J=7.32 \mathrm{~Hz}), 2.37(4 \mathrm{H}, \mathrm{q}, J=7.32 \mathrm{~Hz}), 2.44(3 \mathrm{H}, \mathrm{s}), 3.02(2 \mathrm{H}$, $\mathrm{d}, J=3.66 \mathrm{~Hz}), 3.79(2 \mathrm{H}, \mathrm{d}, J=5.12 \mathrm{~Hz}), 5.50-5.64(2 \mathrm{H}, \mathrm{m})$, $7.34(2 \mathrm{H}, \mathrm{d}, J=8.05 \mathrm{~Hz}), 7.72(2 \mathrm{H}, \mathrm{d}, J=8.05 \mathrm{~Hz}) .{ }^{1} \mathrm{HNMR}$ $\left(\mathrm{C}_{6} \mathrm{D}_{6}\right) \delta 0.83(6 \mathrm{H}, \mathrm{t}, J=7.08 \mathrm{~Hz}), 1.87(3 \mathrm{H}, \mathrm{s}), 2.21(4 \mathrm{H}, \mathrm{q}$, $J=7.08 \mathrm{~Hz}), 2.75(2 \mathrm{H}, \mathrm{d}, J=5.86 \mathrm{~Hz}), 3.41(2 \mathrm{H}, \mathrm{d}, J=$ $7.32 \mathrm{~Hz}), 5.33(1 \mathrm{H}, \mathrm{dt}, J=15.61,5.86 \mathrm{~Hz}), 5.48(1 \mathrm{H}, \mathrm{dt}, J=$ $15.61,7.32 \mathrm{~Hz}), 6.76(2 \mathrm{H}, \mathrm{d}, J=7.81 \mathrm{~Hz}), 7.69(2 \mathrm{H}, \mathrm{d}, J=$ $7.81 \mathrm{~Hz})$. HRMS $\left(\mathrm{FAB}^{+}\right)(\mathrm{M}+\mathrm{H})^{+}$, Found: $m / z 282.15198$. Calcd for $\mathrm{C}_{15} \mathrm{H}_{24} \mathrm{NO}_{2} \mathrm{~S}$ : 282.15277 .

$N, N$-Dipropyl-4-tosyl-2-butenylamine (3e): An oil $(\mathrm{Z} / \mathrm{E}=$ $85 / 15$ ). IR (neat) 3030, 2958, 2932, 2871, 2804, 1597, 1458, 1404, 1381, 1318, 1303, 1239, 1185, 1141, 1088, 1020, 976, $889,816,712,688 \mathrm{~cm}^{-1} . \mathrm{Z}$ isomer; ${ }^{1} \mathrm{HNMR}\left(\mathrm{CDCl}_{3}\right) \delta 0.81$ $(6 \mathrm{H}, \mathrm{t}, J=7.32 \mathrm{~Hz}), 1.33(4 \mathrm{H}, \mathrm{sext}, J=7.32 \mathrm{~Hz}), 2.18(4 \mathrm{H}, \mathrm{t}$, $J=7.32 \mathrm{~Hz}), 2.44(3 \mathrm{H}, \mathrm{s}), 2.74(2 \mathrm{H}, \mathrm{dd}, J=6.34,1.71 \mathrm{~Hz})$, 
$3.89(2 \mathrm{H}, \mathrm{d}, J=8.05 \mathrm{~Hz}), 5.52(1 \mathrm{H}, \mathrm{dtt}, J=10.98,8.05$, $1.71 \mathrm{~Hz}), 5.83(1 \mathrm{H}, \quad \mathrm{dt}, \quad J=10.98,6.34 \mathrm{~Hz}), 7.34(2 \mathrm{H}, \mathrm{d}$, $J=8.05 \mathrm{~Hz}), 7.75(2 \mathrm{H}, \mathrm{d}, J=8.05 \mathrm{~Hz})$. E isomer; ${ }^{1} \mathrm{HNMR}$ $\left(\mathrm{CDCl}_{3}\right) \delta 0.83(6 \mathrm{H}, \mathrm{t}, J=7.32 \mathrm{~Hz}), 1.39(4 \mathrm{H}$, sext, $J=$ $7.32 \mathrm{~Hz}), 2.27(4 \mathrm{H}, \mathrm{t}, J=7.32 \mathrm{~Hz}), 2.44(3 \mathrm{H}, \mathrm{s}), 3.02(2 \mathrm{H}, \mathrm{d}$, $J=4.88 \mathrm{~Hz}), 3.78(2 \mathrm{H}, \mathrm{d}, J=6.10 \mathrm{~Hz}), 5.53-5.60(1 \mathrm{H}, \mathrm{m})$, $5.61(1 \mathrm{H}, \mathrm{dt}, J=15.37,4.88 \mathrm{~Hz}), 7.33(2 \mathrm{H}, \mathrm{d}, J=8.05 \mathrm{~Hz})$, $7.73(2 \mathrm{H}, \mathrm{d}, J=8.05 \mathrm{~Hz})$. HRMS $\left(\mathrm{FAB}^{+}\right)(\mathrm{M}+\mathrm{H})^{+}$, Found: $m / z$ 310.18535. Calcd for $\mathrm{C}_{17} \mathrm{H}_{28} \mathrm{NO}_{2} \mathrm{~S}: 310.18407$.

$N, N$-Dibutyl-4-tosyl-2-butenylamine (3g): $\quad$ An oil $(\mathrm{Z} / \mathrm{E}=$ 87/13). IR (neat) 3030, 2955, 2930, 2871, 2803, 1598, 1495, 1457, 1404, 1378, 1318, 1303, 1234, 1140, 1088, 1019, 974, $887,815,712,687 \mathrm{~cm}^{-1} . \mathrm{Z}$ isomer; ${ }^{1} \mathrm{HNMR}\left(\mathrm{CDCl}_{3}\right) \delta 0.88$ $(6 \mathrm{H}, \mathrm{t}, J=7.31 \mathrm{~Hz}), 1.20-1.40(8 \mathrm{H}, \mathrm{m}), 2.20(4 \mathrm{H}, \mathrm{t}, J=$ $7.81 \mathrm{~Hz}), 2.44(3 \mathrm{H}, \mathrm{s}), 2.72(2 \mathrm{H}, \mathrm{dd}, J=6.34,1.71 \mathrm{~Hz}), 3.89$ $(2 \mathrm{H}, \mathrm{d}, J=7.81 \mathrm{~Hz}), 5.52(1 \mathrm{H}, \mathrm{dtt}, J=10.98,7.81,1.71 \mathrm{~Hz})$, $5.82(1 \mathrm{H}, \mathrm{dt}, J=10.98,6.34 \mathrm{~Hz}), 7.33(2 \mathrm{H}, \mathrm{d}, J=8.05 \mathrm{~Hz})$, $7.76(2 \mathrm{H}, \mathrm{d}, J=8.05 \mathrm{~Hz})$. E isomer; ${ }^{1} \mathrm{HNMR}\left(\mathrm{CDCl}_{3}\right) \delta 0.89$ $(6 \mathrm{H}, \mathrm{t}, \quad J=7.07 \mathrm{~Hz}), 1.20-1.40(8 \mathrm{H}, \mathrm{m}), 2.29(4 \mathrm{H}, \mathrm{t}, J=$ $7.56 \mathrm{~Hz}), 2.44(3 \mathrm{H}, \mathrm{s}), 3.01(2 \mathrm{H}, \mathrm{d}, J=4.64 \mathrm{~Hz}), 3.78(2 \mathrm{H}, \mathrm{d}$, $J=6.10 \mathrm{~Hz}), 5.48-5.56(1 \mathrm{H}, \mathrm{m}), 5.61(1 \mathrm{H}, \mathrm{dt}, J=15.37$, $4.64 \mathrm{~Hz}), 7.32(2 \mathrm{H}, \mathrm{d}, J=8.42 \mathrm{~Hz}), 7.73(2 \mathrm{H}, \mathrm{d}, J=8.42 \mathrm{~Hz})$. HRMS $\left(\mathrm{FAB}^{+}\right)(\mathrm{M}+\mathrm{H})^{+}$, Found: $m / z$ 338.21518. Calcd for $\mathrm{C}_{19} \mathrm{H}_{32} \mathrm{NO}_{2} \mathrm{~S}: 338.21537$.

$\boldsymbol{N}$-Butyl- $\boldsymbol{N}$-methyl-4-tosyl-2-butenylamine (3h): An oil $(\mathrm{Z} / \mathrm{E}=72 / 28)$. IR (neat) 3030, 2956, 2931, 2871, 2789, 1597, $1494,1458,1403,1376,1318,1303,1240,1206,1144,1088$, $1040,1019,976,886,859,816,782,713,688,664 \mathrm{~cm}^{-1} . \mathrm{Z}$ isomer; ${ }^{1} \mathrm{HNMR}\left(\mathrm{CDCl}_{3}\right) \delta 0.89(3 \mathrm{H}, \mathrm{t}, J=7.32 \mathrm{~Hz}), 1.22-1.44$ $(4 \mathrm{H}, \mathrm{m}), 2.02(3 \mathrm{H}, \mathrm{s}), 2.16(2 \mathrm{H}, \mathrm{t}, J=7.81 \mathrm{~Hz}), 2.44(3 \mathrm{H}, \mathrm{s})$, $2.70(2 \mathrm{H}, \mathrm{dd}, J=6.34,1.46 \mathrm{~Hz}), 3.90(2 \mathrm{H}, \mathrm{d}, J=7.81 \mathrm{~Hz})$, $5.54(1 \mathrm{H}, \mathrm{dtt}, J=10.98,7.81,1.46 \mathrm{~Hz}), 5.83(1 \mathrm{H}, \mathrm{dt}, J=$ $10.98,6.34 \mathrm{~Hz}), 7.34(2 \mathrm{H}, \mathrm{d}, J=8.05 \mathrm{~Hz}), 7.75(2 \mathrm{H}, \mathrm{d}, J=$ $8.05 \mathrm{~Hz})$. E isomer; ${ }^{1} \mathrm{HNMR}\left(\mathrm{CDCl}_{3}\right) \delta 0.90(3 \mathrm{H}, \mathrm{t}, J=$ $7.32 \mathrm{~Hz}), 1.22-1.44(4 \mathrm{H}, \mathrm{m}), 2.08(3 \mathrm{H}, \mathrm{s}), 2.24(2 \mathrm{H}, \mathrm{t}, J=$ $7.81 \mathrm{~Hz}), 2.44(3 \mathrm{H}, \mathrm{s}), 2.94(2 \mathrm{H}, \mathrm{d}, J=4.64 \mathrm{~Hz}), 3.79(2 \mathrm{H}, \mathrm{d}$, $J=5.37 \mathrm{~Hz}), \quad 5.52-5.62(1 \mathrm{H}, \mathrm{m}), 5.61(1 \mathrm{H}, \mathrm{dt}, J=15.38$, $5.37 \mathrm{~Hz}), 7.33(2 \mathrm{H}, \mathrm{d}, J=7.81 \mathrm{~Hz}), 7.73(2 \mathrm{H}, \mathrm{d}, J=7.81 \mathrm{~Hz})$. HRMS $\left(\mathrm{FAB}^{+}\right)(\mathrm{M}+\mathrm{H})^{+}$, Found: $m / z$ 296.16882. Calcd for $\mathrm{C}_{16} \mathrm{H}_{26} \mathrm{NO}_{2} \mathrm{~S}$ : 296.16842 .

$\boldsymbol{N}$-Isopropyl- $\boldsymbol{N}$-methyl-4-tosyl-2-butenylamine (3i): An oil $(\mathrm{Z} / \mathrm{E}=80 / 20)$. IR (neat) 3030, 2966, 2792, 1597, 1453, 1402, $1383,1362,1317,1304,1236,1143,1088,1018,975,876,816$, $769,712,688,664 \mathrm{~cm}^{-1} . \mathrm{Z}$ isomer; ${ }^{1} \mathrm{HNMR}\left(\mathrm{CDCl}_{3}\right) \delta 0.92$ $(6 \mathrm{H}, \mathrm{d}, J=6.58 \mathrm{~Hz}), 1.99(3 \mathrm{H}, \mathrm{s}), 2.44(3 \mathrm{H}, \mathrm{s}), 2.68(1 \mathrm{H}, \mathrm{sept}$, $J=6.58 \mathrm{~Hz}), 2.73(2 \mathrm{H}, \mathrm{dd}, J=6.34,1.22 \mathrm{~Hz}), 3.91(2 \mathrm{H}, \mathrm{d}$, $J=8.05 \mathrm{~Hz}), 5.52(1 \mathrm{H}, \mathrm{dtt}, J=10.98,8.05,1.22 \mathrm{~Hz}), 5.83(1 \mathrm{H}$, $\mathrm{dt}, J=10.98,6.34 \mathrm{~Hz}), 7.35(2 \mathrm{H}, \mathrm{d}, J=8.05 \mathrm{~Hz}), 7.76(2 \mathrm{H}, \mathrm{d}$, $J=8.05 \mathrm{~Hz})$. E isomer; ${ }^{1} \mathrm{HNMR}\left(\mathrm{CDCl}_{3}\right) \delta 0.93(6 \mathrm{H}, \mathrm{d}, J=$ $6.58 \mathrm{~Hz}), 2.04(3 \mathrm{H}, \mathrm{s}), 2.44(3 \mathrm{H}, \mathrm{s}), 2.68(1 \mathrm{H}$, sept, $J=$ $6.57 \mathrm{~Hz}), 2.98(2 \mathrm{H}, \mathrm{d}, J=4.15 \mathrm{~Hz}), 3.78(2 \mathrm{H}, \mathrm{d}, J=5.86 \mathrm{~Hz})$, $5.56-5.62(1 \mathrm{H}, \mathrm{m}), 5.60(1 \mathrm{H}, \mathrm{dt}, J=15.14,5.86 \mathrm{~Hz}), 7.32(2 \mathrm{H}$, $\mathrm{d}, J=8.05 \mathrm{~Hz}), 7.73(2 \mathrm{H}, \mathrm{d}, J=8.05 \mathrm{~Hz}) . \mathrm{HRMS}\left(\mathrm{FAB}^{+}\right)$ $(\mathrm{M}+\mathrm{H})^{+}$, Found: $m / z$ 282.15318. Calcd for $\mathrm{C}_{15} \mathrm{H}_{24} \mathrm{NO}_{2} \mathrm{~S}$ : 282.15277 .

1-(4-Tosyl-2-butenyl)pyrrolidine (3j): An oil $(\mathrm{Z} / \mathrm{E}=44$ / 56). IR (neat) 3030, 2963, 2875, 2789, 1597, 1494, 1459, 1402, 1347, 1317, 1304, 1291, 1236, 1142, 1088, 1018, 969, 929, 878, $816,715,669 \mathrm{~cm}^{-1} . \mathrm{Z}$ isomer; ${ }^{1} \mathrm{HNMR}\left(\mathrm{CDCl}_{3}\right) \delta 1.65-1.78$ $(4 \mathrm{H}, \mathrm{m}), 2.28-2.40(4 \mathrm{H}, \mathrm{m}), 2.44(3 \mathrm{H}, \mathrm{s}), 2.88(2 \mathrm{H}, \mathrm{d}, J=$ $6.59 \mathrm{~Hz}), 3.91(2 \mathrm{H}, \mathrm{d}, J=7.81 \mathrm{~Hz}), 5.51(1 \mathrm{H}, \mathrm{dt}, J=10.98$, $7.81 \mathrm{~Hz}), 5.84(1 \mathrm{H}, \mathrm{dt}, J=10.98,6.59 \mathrm{~Hz}), 7.34(2 \mathrm{H}, \mathrm{d}, J=$
$8.29 \mathrm{~Hz}), 7.76(2 \mathrm{H}, \mathrm{d}, J=8.29 \mathrm{~Hz}) . \mathrm{E}$ isomer; ${ }^{1} \mathrm{HNMR}\left(\mathrm{CDCl}_{3}\right)$ $\delta 1.65-1.80(4 \mathrm{H}, \mathrm{m}), 2.28-2.40(4 \mathrm{H}, \mathrm{m}), 2.44(3 \mathrm{H}, \mathrm{s}), 3.05(2 \mathrm{H}, \mathrm{d}$, $J=4.88 \mathrm{~Hz}), 3.78(2 \mathrm{H}, \mathrm{d}, J=5.86 \mathrm{~Hz}), 5.60(1 \mathrm{H}, \mathrm{dt}, J=15.34$, $5.86 \mathrm{~Hz}), 5.64(1 \mathrm{H}, \mathrm{dt}, J=15.34,4.88 \mathrm{~Hz}), 7.33(2 \mathrm{H}, \mathrm{d}, J=$ $8.29 \mathrm{~Hz}), 7.73(2 \mathrm{H}, \mathrm{d}, J=8.29 \mathrm{~Hz})$. HRMS $\left(\mathrm{FAB}^{+}\right)(\mathrm{M}+\mathrm{H})^{+}$, Found: $m / z$ 280.13644. Calcd for $\mathrm{C}_{15} \mathrm{H}_{22} \mathrm{NO}_{2} \mathrm{~S}$ : 280.13712 .

1-(4-Tosyl-2-butenyl)piperidine (3k): An oil $(\mathrm{Z} / \mathrm{E}=55 /$ 45). IR (neat) 3030, 2933, 2853, 2795, 2754, 1597, 1494, 1467, 1453, 1402, 1372, 1318, 1303, 1242, 1141, 1118, 1088, 1041, 1019, 991, 901, 861, 816, 778, 734, 713, 690, $664 \mathrm{~cm}^{-1} . \mathrm{Z}$ isomer; ${ }^{1} \mathrm{HNMR}\left(\mathrm{CDCl}_{3}\right) \delta 1.30-1.45(2 \mathrm{H}, \mathrm{m}), 1.45-1.55$ (4H, m), 2.10$2.30(4 \mathrm{H}, \mathrm{m}), 2.44(3 \mathrm{H}, \mathrm{s}), 2.98(2 \mathrm{H}, \mathrm{dd}, J=6.59,1.71 \mathrm{~Hz}), 3.90$ $(2 \mathrm{H}, \mathrm{d}, J=7.81 \mathrm{~Hz}), 5.54(1 \mathrm{H}, \mathrm{dtt}, J=10.98,7.81,1.71 \mathrm{~Hz})$, $5.84(1 \mathrm{H}, \mathrm{dt}, J=10.98,6.59 \mathrm{~Hz}), 7.33(2 \mathrm{H}, \mathrm{d}, J=8.29 \mathrm{~Hz})$, $7.72(2 \mathrm{H}, \mathrm{d}, J=8.29 \mathrm{~Hz})$. E isomer; ${ }^{1} \mathrm{HNMR}\left(\mathrm{CDCl}_{3}\right) \delta 1.30$ $1.45(2 \mathrm{H}, \mathrm{m}), 1.40-1.55(4 \mathrm{H}, \mathrm{m}), 2.10-2.30(4 \mathrm{H}, \mathrm{m}), 2.44(3 \mathrm{H}$, s), $2.88(2 \mathrm{H}, \mathrm{d}, J=4.64 \mathrm{~Hz}), 3.79(2 \mathrm{H}, \mathrm{d}, J=5.37 \mathrm{~Hz}), 5.50$ $5.60(1 \mathrm{H}, \mathrm{m}), 5.58(1 \mathrm{H}, \mathrm{dt}, J=15.59,5.37 \mathrm{~Hz}), 7.34(2 \mathrm{H}$, $\mathrm{d}, J=8.29 \mathrm{~Hz}), 7.76(2 \mathrm{H}, \mathrm{d}, J=8.29 \mathrm{~Hz}) . \operatorname{HRMS}\left(\mathrm{FAB}^{+}\right)$ $(\mathrm{M}+\mathrm{H})^{+}$, Found: $m / z$ 294.15420. Calcd for $\mathrm{C}_{16} \mathrm{H}_{24} \mathrm{NO}_{2} \mathrm{~S}$ : 294.15277.

Nucleophilic Addition Reaction of Diethylamine (2d) to $(E)$ 1-Tosyl-1,3-butadiene (1) under Dilute Conditions at $60^{\circ} \mathrm{C}$ (Table 3, Entry 5). To a solution of (E)-1-tosyl-1,3-butadiene (1) $(129 \mathrm{mg}, 0.62 \mathrm{mmol})$ in THF $(198 \mathrm{~mL})$ was added diethylamine (2d) $(0.77 \mathrm{~mL}, 7.43 \mathrm{mmol})$ at $60^{\circ} \mathrm{C}$ under a nitrogen atmosphere. After stirring for $72 \mathrm{~h}$, the reaction mixture was quenched by adding silica gel (3.34 g), and the solvent was evaporated. The residue was purified by column chromatography $\left(\mathrm{SiO}_{2}\right.$, hexane/ $\mathrm{EtOAc}=5 / 1, \mathrm{v} / \mathrm{v}, \mathrm{EtOAc}$, then $\mathrm{EtOAc} / \mathrm{MeOH}=3 / 1, \mathrm{v} / \mathrm{v}$ ) to give a mixture of $(Z)$ - and $(E)-3 d(125 \mathrm{mg}, 72 \%, \mathrm{Z} / \mathrm{E}=98 / 2)$ and to recover unreacted $\mathbf{1}(36 \mathrm{mg}, 28 \%)$.

A Representative Procedure for the Nucleophilic Addition Reaction of Piperidine (2k) to Ethyl $(E)$-2,4-Pentadienoate (4) (Table 5, Entry 10). To a solution of ethyl (E)-2,4-pentadienoate $(4)^{22}(67 \mathrm{mg}, 0.53 \mathrm{mmol})$ in THF $(5.3 \mathrm{~mL})$ was added piperidine $(\mathbf{2 k})(0.079 \mathrm{~mL}, 0.80 \mathrm{mmol})$ at $25^{\circ} \mathrm{C}$ under a nitrogen atmosphere. After stirring for $72 \mathrm{~h}$, the reaction mixture was quenched by adding silica gel ( $2.80 \mathrm{~g}$ ), and the solvent was evaporated. The residue was purified by column chromatography $\left(\mathrm{SiO}_{2}\right.$, hexane $/ \mathrm{EtOAc}=5 / 1, \mathrm{v} / \mathrm{v}, \mathrm{EtOAc}$, then EtOAc $/ \mathrm{MeOH}=3 / 1$, $\mathrm{v} / \mathrm{v})$ to give a mixture of $(Z)-$ and $(E)-5 \mathbf{k}(65 \mathrm{mg}, 58 \%, \mathrm{Z} / \mathrm{E}=$ 68/32) and to recover unreacted 4 (25 mg, 37\%).

In a similar way, reactions of amines $\mathbf{2 a}-\mathbf{2 e}$ and $\mathbf{2} \mathbf{g}-\mathbf{2} \mathbf{j}$ with ethyl (E)-2,4-pentadienoate (4) were carried out to give the corresponding addition products $\mathbf{5 a}-\mathbf{5 e}$ and $\mathbf{5 g}-\mathbf{5 j}$. The physical and spectral data of $\mathbf{5 a - 5 e}$ and $\mathbf{5} \mathbf{g}-\mathbf{5} \mathbf{k}$ and by-products are given in the following. $\mathrm{Z} / \mathrm{E}$ ratios were determined by $400 \mathrm{MHz}{ }^{1} \mathrm{HNMR}$ spectra. In the cases of $\mathrm{Me}_{2} \mathrm{NH}, \mathrm{Et}_{2} \mathrm{NH}, n-\mathrm{Pr}_{2} \mathrm{NH}, n-\mathrm{Bu}_{2} \mathrm{NH}$, $n-\mathrm{Bu}(\mathrm{Me}) \mathrm{NH}, i-\mathrm{Pr}(\mathrm{Me}) \mathrm{NH}$, and pyrrolidine, the $\mathrm{Z} / \mathrm{E}$ ratios were measured in $\mathrm{CDCl}_{3}$ containing $\mathrm{CF}_{3} \mathrm{CO}_{2} \mathrm{D}$ in order to separate ${ }^{1} \mathrm{H}$ NMR peaks, and no isomerization was observed for $24 \mathrm{~h}$. Only specific chemical shifts, which were measured in $\mathrm{CDCl}_{3}$ containing $\mathrm{CF}_{3} \mathrm{CO}_{2} \mathrm{D}$ and used for determination of $\mathrm{Z} / \mathrm{E}$ ratios, are shown.

Ethyl 5-(Propylamino)-3-pentenoate (5a): An oil (Z/ $\mathrm{E}=7 / 93$ ). IR (neat) 3317, 2960, 2933, 2874, 2816, 1738, 1652, 1462, 1408, 1369, 1330, 1301, 1256, 1175, 1096, 1029, 972, 937, 856, 808, 786, $751 \mathrm{~cm}^{-1} . \mathrm{Z}$ isomer; ${ }^{1} \mathrm{HNMR}\left(\mathrm{CDCl}_{3}\right)$ $\delta 0.92(3 \mathrm{H}, \mathrm{t}, J=7.32 \mathrm{~Hz}), 1.26(3 \mathrm{H}, \mathrm{t}, J=7.07 \mathrm{~Hz}), 1.52$ $(2 \mathrm{H}$, sext, $J=7.32 \mathrm{~Hz}), 2.10-2.25(1 \mathrm{H}, \mathrm{m}), 2.59(2 \mathrm{H}, \mathrm{t}, J=$ $7.32 \mathrm{~Hz}), 3.12(2 \mathrm{H}, \mathrm{d}, J=5.12 \mathrm{~Hz}), 3.30(2 \mathrm{H}, \mathrm{d}, J=4.64 \mathrm{~Hz})$, 
$4.15(2 \mathrm{H}, \mathrm{q}, J=7.07 \mathrm{~Hz}), 5.72-5.78(2 \mathrm{H}, \mathrm{m})$. E isomer; ${ }^{1} \mathrm{H} \mathrm{NMR}$ $\left(\mathrm{CDCl}_{3}\right) \delta 0.92(3 \mathrm{H}, \mathrm{t}, J=7.32 \mathrm{~Hz}), 1.26(3 \mathrm{H}, \mathrm{t}, J=7.07 \mathrm{~Hz})$, $1.52(2 \mathrm{H}$, sext, $J=7.32 \mathrm{~Hz}), 2.10-2.25(1 \mathrm{H}, \mathrm{m}), 2.59(2 \mathrm{H}, \mathrm{t}, J=$ $7.32 \mathrm{~Hz}), 3.06(2 \mathrm{H}, \mathrm{d}, J=5.85 \mathrm{~Hz}), 3.26(2 \mathrm{H}, \mathrm{d}, J=5.37 \mathrm{~Hz})$, $4.14(2 \mathrm{H}, \mathrm{q}, J=7.07 \mathrm{~Hz}), 5.67(1 \mathrm{H}, \mathrm{dt}, J=15.61,5.37 \mathrm{~Hz})$, $5.74(1 \mathrm{H}, \mathrm{dt}, J=15.61,5.85 \mathrm{~Hz})$. HRMS $\left(\mathrm{FAB}^{+}\right)(\mathrm{M}+\mathrm{H})^{+}$, Found: $m / z$ 186.14923. Calcd for $\mathrm{C}_{10} \mathrm{H}_{20} \mathrm{NO}_{2}$ : 186.14940 .

1-Propyl-3,6-dihydro-1H-pyridin-2-one: An oil. IR (neat) 2964, 2931, 2872, 1637, 1497, 1458, 1411, 1267, 1164, 979, $900,675 \mathrm{~cm}^{-1} .{ }^{1} \mathrm{HNMR}\left(\mathrm{CDCl}_{3}\right) \delta 0.92(3 \mathrm{H}, \mathrm{t}, J=7.32 \mathrm{~Hz})$, $1.61(2 \mathrm{H}$, sext, $J=7.32 \mathrm{~Hz}), 2.90-2.98(2 \mathrm{H}, \mathrm{m}), 3.39(2 \mathrm{H}, \mathrm{t}$, $J=7.32 \mathrm{~Hz}), 3.88-3.93(2 \mathrm{H}, \mathrm{m}), 5.66-5.80(2 \mathrm{H}, \mathrm{m})$. HRMS $\left(\mathrm{FAB}^{+}\right)(\mathrm{M}+\mathrm{H})^{+}$, Found: $m / z$ 140.10772. Calcd for $\mathrm{C}_{8} \mathrm{H}_{14} \mathrm{NO}$ : 140.10754.

Ethyl 5-(Butylamino)-3-pentenoate (5b): An oil $(\mathrm{Z} / \mathrm{E}=$ 11/89). IR (neat) 3391, 2959, 2931, 2872, 2821, 1736, 1652, 1540, 1465, 1409, 1370, 1303, 1254, 1174, 1096, 1029, 973 , 940, 856, 810, $736 \mathrm{~cm}^{-1} . \mathrm{Z}$ isomer; ${ }^{1} \mathrm{HNMR}\left(\mathrm{CDCl}_{3}\right) \delta 0.91$ $(3 \mathrm{H}, \mathrm{t}, J=7.32 \mathrm{~Hz}), 1.26(3 \mathrm{H}, \mathrm{t}, J=7.07 \mathrm{~Hz}), 1.34(2 \mathrm{H}, \mathrm{sext}$, $J=7.32 \mathrm{~Hz}), 1.48(2 \mathrm{H}$, quint, $J=7.32 \mathrm{~Hz}), 1.90-2.02(1 \mathrm{H}, \mathrm{m})$, $2.62(2 \mathrm{H}, \mathrm{t}, J=7.32 \mathrm{~Hz}), 3.12(2 \mathrm{H}, \mathrm{d}, J=5.37 \mathrm{~Hz}), 3.30(2 \mathrm{H}$, $\mathrm{d}, J=4.88 \mathrm{~Hz}), 4.15(2 \mathrm{H}, \mathrm{q}, J=7.07 \mathrm{~Hz}), 5.66-5.77(2 \mathrm{H}, \mathrm{m})$. E isomer; ${ }^{1} \mathrm{HNMR}\left(\mathrm{CDCl}_{3}\right) \delta 0.91(3 \mathrm{H}, \mathrm{t}, J=7.32 \mathrm{~Hz}), 1.26$ $(3 \mathrm{H}, \mathrm{t}, J=7.07 \mathrm{~Hz}), 1.34(2 \mathrm{H}, \operatorname{sext}, J=7.32 \mathrm{~Hz}), 1.48(2 \mathrm{H}$, quint, $J=7.32 \mathrm{~Hz}), 1.90-2.02(1 \mathrm{H}, \mathrm{m}), 2.61(2 \mathrm{H}, \mathrm{t}, J=$ $7.32 \mathrm{~Hz}), 3.06(2 \mathrm{H}, \mathrm{d}, J=5.61 \mathrm{~Hz}), 3.25(2 \mathrm{H}, \mathrm{d}, J=5.12 \mathrm{~Hz})$, $4.14(2 \mathrm{H}, \mathrm{q}, J=7.07 \mathrm{~Hz}), 5.67(1 \mathrm{H}, \mathrm{dt}, J=15.61,5.12 \mathrm{~Hz})$, $5.73(1 \mathrm{H}, \mathrm{dt}, J=15.61,5.61 \mathrm{~Hz})$. HRMS $\left(\mathrm{FAB}^{+}\right)(\mathrm{M}+\mathrm{H})^{+}$, Found: $m / z$ 200.16450. Calcd for $\mathrm{C}_{11} \mathrm{H}_{22} \mathrm{NO}_{2}$ : 200.16505.

1-Butyl-3,6-dihydro-1H-pyridin-2-one: An oil. IR (neat) 3047, 2958, 2931, 2871, 1634, 1496, 1456, 1411, 1375, 1326, $1298,1251,1197,1165,1131,1112,1083,984,854,734$, $677 \mathrm{~cm}^{-1} .{ }^{1} \mathrm{HNMR}\left(\mathrm{CDCl}_{3}\right) \delta 0.94(3 \mathrm{H}, \mathrm{t}, J=7.56 \mathrm{~Hz}), 1.34$ $(2 \mathrm{H}$, sext, $J=7.56 \mathrm{~Hz}), 1.56(2 \mathrm{H}$, quint, $J=7.56 \mathrm{~Hz}), 2.93-$ $2.97(2 \mathrm{H}, \mathrm{m}), 3.42(2 \mathrm{H}, \mathrm{t}, J=7.56 \mathrm{~Hz}), 3.88-3.94(2 \mathrm{H}, \mathrm{m})$, 5.69-5.80 (2H, m). HRMS $\left(\mathrm{FAB}^{+}\right)(\mathrm{M}+\mathrm{H})^{+}$, Found: $m / z$ 154.12298. Calcd for $\mathrm{C}_{9} \mathrm{H}_{16} \mathrm{NO}$ : 154.12319.

Ethyl 5-(Dimethylamino)-3-pentenoate (5c): An oil (Z/E = 76/24). IR (neat) 2979, 2941, 2857, 2817, 2772, 1737, 1652, $1457,1367,1320,1259,1175,1096,1031,975,853 \mathrm{~cm}^{-1} . \mathrm{Z}$ isomer; ${ }^{1} \mathrm{H} \mathrm{NMR}\left(\mathrm{CDCl}_{3}\right) \delta 1.26(3 \mathrm{H}, \mathrm{t}, J=7.07 \mathrm{~Hz}), 2.24(6 \mathrm{H}, \mathrm{s})$, $2.96(2 \mathrm{H}, \mathrm{d}, J=6.34 \mathrm{~Hz}), 3.13(2 \mathrm{H}, \mathrm{d}, J=6.42 \mathrm{~Hz}), 4.15(2 \mathrm{H}, \mathrm{q}$, $J=7.07 \mathrm{~Hz}), 5.67(1 \mathrm{H}, \mathrm{dt}, J=10.98,6.34 \mathrm{~Hz}), 5.75(1 \mathrm{H}, \mathrm{dt}$, $J=10.98,6.42 \mathrm{~Hz}) .{ }^{1} \mathrm{HNMR}\left(\mathrm{CDCl}_{3}\right.$ with $\left.\mathrm{CF}_{3} \mathrm{CO}_{2} \mathrm{D}\right) \delta 3.74-$ $3.82(2 \mathrm{H}, \mathrm{m})$. E isomer; ${ }^{1} \mathrm{HNMR}\left(\mathrm{CDCl}_{3}\right) \delta 1.26(3 \mathrm{H}, \mathrm{t}$, $J=7.07 \mathrm{~Hz}), 2.23(6 \mathrm{H}, \mathrm{s}), 2.92(2 \mathrm{H}, \mathrm{d}, J=6.34 \mathrm{~Hz}), 3.07(2 \mathrm{H}$, $\mathrm{d}, J=6.59 \mathrm{~Hz}), 4.14(2 \mathrm{H}, \mathrm{q}, J=7.07 \mathrm{~Hz}), 5.61(1 \mathrm{H}, \mathrm{dt}$, $J=15.37,6.34 \mathrm{~Hz}), 5.72(1 \mathrm{H}, \mathrm{dt}, J=15.37,6.59 \mathrm{~Hz}) .{ }^{1} \mathrm{H} \mathrm{NMR}$ $\left(\mathrm{CDCl}_{3}\right.$ with $\left.\mathrm{CF}_{3} \mathrm{CO}_{2} \mathrm{D}\right) \delta$ 3.63-3.71 $(2 \mathrm{H}, \mathrm{m}) . \mathrm{HRMS}\left(\mathrm{FAB}^{+}\right)$ $(\mathrm{M}+\mathrm{H})^{+}$, Found: $m / z$ 172.13432. Calcd for $\mathrm{C}_{9} \mathrm{H}_{18} \mathrm{NO}_{2}$ : 172.13375 .

Ethyl 5-(Diethylamino)-3-pentenoate (5d): An oil (Z/E = 87/13). IR (neat) 2960, 2923, 2853, 1732, 1457, 1367, 1280, $1260,1181,1033,850,705,670 \mathrm{~cm}^{-1} . \mathrm{Z}$ isomer; ${ }^{1} \mathrm{HNMR}\left(\mathrm{C}_{6} \mathrm{D}_{6}\right)$ $\delta 0.90(6 \mathrm{H}, \mathrm{t}, J=7.08 \mathrm{~Hz}), 0.93(3 \mathrm{H}, \mathrm{t}, J=7.07 \mathrm{~Hz}), 2.35(4 \mathrm{H}, \mathrm{q}$, $J=7.08 \mathrm{~Hz}), 2.95-2.98(4 \mathrm{H}, \mathrm{m}), 3.92(2 \mathrm{H}, \mathrm{q}, J=7.07 \mathrm{~Hz}), 5.66$ $(1 \mathrm{H}, \mathrm{dtt}, J=10.98,6.59,1.46 \mathrm{~Hz}), 5.78(1 \mathrm{H}, \mathrm{dtt}, J=10.98,7.32$, $1.46 \mathrm{~Hz}) .{ }^{1} \mathrm{HNMR}\left(\mathrm{CDCl}_{3}\right.$ with $\left.\mathrm{CF}_{3} \mathrm{CO}_{2} \mathrm{D}\right) \delta 2.97(2 \mathrm{H}, \mathrm{d}$, $J=6.59 \mathrm{~Hz})$. E isomer; ${ }^{1} \mathrm{HNMR}\left(\mathrm{C}_{6} \mathrm{D}_{6}\right) \delta 0.94(9 \mathrm{H}, \mathrm{t}, J=$ $7.08 \mathrm{~Hz}), 2.39(4 \mathrm{H}, \mathrm{q}, J=7.08 \mathrm{~Hz}), 2.87(2 \mathrm{H}, \mathrm{dd}, J=6.83$, $1.22 \mathrm{~Hz}), 2.94(2 \mathrm{H}, \mathrm{dd}, J=6.34,1.22 \mathrm{~Hz}), 3.92(2 \mathrm{H}, \mathrm{q}$, $J=7.08 \mathrm{~Hz}), 5.56(1 \mathrm{H}, \mathrm{dtt}, J=15.37,6.34,1.22 \mathrm{~Hz}), 5.73(1 \mathrm{H}$, $\mathrm{dtt}, J=15.37,6.83,1.22 \mathrm{~Hz}) .{ }^{1} \mathrm{HNMR}\left(\mathrm{CDCl}_{3}\right.$ with $\left.\mathrm{CF}_{3} \mathrm{CO}_{2} \mathrm{D}\right)$ $\delta 2.83(2 \mathrm{H}, \mathrm{d}, J=7.07 \mathrm{~Hz})$. HRMS $\left(\mathrm{FAB}^{+}\right)(\mathrm{M}+\mathrm{H})^{+}$, Found: $m / z$ 200.16450. Calcd for $\mathrm{C}_{11} \mathrm{H}_{22} \mathrm{NO}_{2}: 200.16505$.

Ethyl 5-(Dipropylamino)-3-pentenoate (5e): An oil (Z/ $\mathrm{E}=90 / 10$ ). IR (neat) 3030, 2959, 2934, 2873, 2800, 1740, 1458, 1367, 1319, 1254, 1163, 1095, 1075, 1033, 973, 848, 748, $676 \mathrm{~cm}^{-1}$. Z isomer; ${ }^{1} \mathrm{HNMR}\left(\mathrm{CDCl}_{3}\right) \delta 0.87(6 \mathrm{H}, \mathrm{t}, J=$ $7.32 \mathrm{~Hz}), 1.26(3 \mathrm{H}, \quad \mathrm{t}, \quad J=7.32 \mathrm{~Hz}), 1.47(4 \mathrm{H}, \quad$ sext, $J=$ $7.32 \mathrm{~Hz}), 2.39(4 \mathrm{H}, \mathrm{t}, J=7.32 \mathrm{~Hz}), 3.12(2 \mathrm{H}, \mathrm{d}, J=5.61 \mathrm{~Hz})$, $3.13(2 \mathrm{H}, \mathrm{d}, J=4.64 \mathrm{~Hz}), 4.15(2 \mathrm{H}, \mathrm{q}, J=7.32 \mathrm{~Hz}), 5.68(1 \mathrm{H}$, $\mathrm{dt}, \quad J=10.98,4.64 \mathrm{~Hz}), 5.73(1 \mathrm{H}, \mathrm{dt}, J=10.98,5.61 \mathrm{~Hz})$. ${ }^{1} \mathrm{HNMR}\left(\mathrm{CDCl}_{3}\right.$ with $\left.\mathrm{CF}_{3} \mathrm{CO}_{2} \mathrm{D}\right) \delta 3.84(2 \mathrm{H}, \mathrm{d}, J=4.64 \mathrm{~Hz})$. E isomer; ${ }^{1} \mathrm{HNMR}\left(\mathrm{CDCl}_{3}\right) \delta 0.87(6 \mathrm{H}, \mathrm{t}, J=7.32 \mathrm{~Hz}), 1.26$ $(3 \mathrm{H}, \mathrm{t}, J=7.32 \mathrm{~Hz}), 1.47(4 \mathrm{H}, \operatorname{sext}, J=7.32 \mathrm{~Hz}), 2.42(4 \mathrm{H}, \mathrm{t}$, $J=7.32 \mathrm{~Hz}), 3.07(4 \mathrm{H}, \mathrm{d}, J=6.34 \mathrm{~Hz}), 4.14(2 \mathrm{H}, \mathrm{q}, J=$ $7.32 \mathrm{~Hz}), 5.63(1 \mathrm{H}, \mathrm{dt}, J=15.61,6.34 \mathrm{~Hz}), 5.66(1 \mathrm{H}, \mathrm{dt}, J=$ 15.61, $6.34 \mathrm{~Hz}) .{ }^{1} \mathrm{HNMR}\left(\mathrm{CDCl}_{3}\right.$ with $\left.\mathrm{CF}_{3} \mathrm{CO}_{2} \mathrm{D}\right) \quad \delta \quad 3.75$ $(2 \mathrm{H}, \mathrm{d}, J=6.34 \mathrm{~Hz})$. HRMS $\left(\mathrm{FAB}^{+}\right)(\mathrm{M}+\mathrm{H})^{+}$, Found: $m / z$ 228.19671. Calcd for $\mathrm{C}_{13} \mathrm{H}_{26} \mathrm{NO}_{2}: 228.19635$.

Ethyl 5-(Dibutylamino)-3-pentenoate (5g): An oil (Z/E = 94/6). IR (neat) 3030, 2957, 2932, 2871, 2798, 1740, 1652, 1456, 1367, 1318, 1252, 1162, 1096, 1034, 973, 937, 852, 735, $668 \mathrm{~cm}^{-1}$. Z isomer; ${ }^{1} \mathrm{HNMR}\left(\mathrm{CDCl}_{3}\right) \delta 0.90(6 \mathrm{H}, \mathrm{t}, J=$ $7.07 \mathrm{~Hz}), \quad 1.26(3 \mathrm{H}, \quad \mathrm{t}, \quad J=7.07 \mathrm{~Hz}), 1.29(4 \mathrm{H}$, sext, $J=$ $7.07 \mathrm{~Hz}), 1.43(4 \mathrm{H}, \mathrm{tt}, J=7.56,7.07 \mathrm{~Hz}), 2.41(4 \mathrm{H}, \mathrm{t}, J=$ $7.56 \mathrm{~Hz}), 3.09(2 \mathrm{H}, \mathrm{d}, J=5.86 \mathrm{~Hz}), 3.12(2 \mathrm{H}, \mathrm{d}, J=5.61 \mathrm{~Hz})$, $4.15(2 \mathrm{H}, \mathrm{q}, J=7.07 \mathrm{~Hz}), 5.68(1 \mathrm{H}, \mathrm{dt}, J=10.73,5.86 \mathrm{~Hz})$, $5.72(1 \mathrm{H}, \quad \mathrm{dt}, \quad J=10.73,5.61 \mathrm{~Hz}) .{ }^{1} \mathrm{HNMR}\left(\mathrm{CDCl}_{3}\right.$ with $\left.\mathrm{CF}_{3} \mathrm{CO}_{2} \mathrm{D}\right) \delta 3.85(2 \mathrm{H}, \mathrm{d}, J=5.61 \mathrm{~Hz}) . \mathrm{E}$ isomer; ${ }^{1} \mathrm{HNMR}$ $\left(\mathrm{CDCl}_{3}\right) \delta 0.90(6 \mathrm{H}, \mathrm{t}, J=7.07 \mathrm{~Hz}), 1.26(3 \mathrm{H}, \mathrm{t}, J=7.07 \mathrm{~Hz})$, $1.27(4 \mathrm{H}$, sext, $J=7.07 \mathrm{~Hz}), 1.43(4 \mathrm{H}, \mathrm{tt}, J=7.32,7.07 \mathrm{~Hz})$, $2.40(4 \mathrm{H}, \mathrm{t}, J=7.32 \mathrm{~Hz}), 3.06(4 \mathrm{H}, \mathrm{d}, J=5.61 \mathrm{~Hz}), 4.13(2 \mathrm{H}$, q, $J=7.07 \mathrm{~Hz}), 5.62(1 \mathrm{H}, \mathrm{dt}, J=15.61,5.61 \mathrm{~Hz}), 5.68(1 \mathrm{H}, \mathrm{dt}$, $J=15.61,5.61 \mathrm{~Hz}) .{ }^{1} \mathrm{HNMR}\left(\mathrm{CDCl}_{3}\right.$ with $\left.\mathrm{CF}_{3} \mathrm{CO}_{2} \mathrm{D}\right) \delta 3.76$ $(2 \mathrm{H}, \mathrm{d}, J=5.61 \mathrm{~Hz})$. HRMS $\left(\mathrm{FAB}^{+}\right)(\mathrm{M}+\mathrm{H})^{+}$, Found: $m / z$ 256.22696. Calcd for $\mathrm{C}_{15} \mathrm{H}_{30} \mathrm{NO}_{2}: 256.22765$.

Ethyl 5-(Butylmethylamino)-3-pentenoate (5h): An oil, $(\mathrm{Z} / \mathrm{E}=74 / 26) . \mathrm{IR}$ (neat) 3028, 2957, 2933, 2872, 2840, 2787, $1739,1652,1462,1367,1319,1255,1164,1096,1063,1033$, $973,858,736 \mathrm{~cm}^{-1} . \mathrm{Z}$ isomer; ${ }^{1} \mathrm{HNMR}\left(\mathrm{CDCl}_{3}\right) \delta 0.91(3 \mathrm{H}, \mathrm{t}$, $J=7.32 \mathrm{~Hz}), 1.26(3 \mathrm{H}, \mathrm{t}, J=7.07 \mathrm{~Hz}), 1.31(2 \mathrm{H}$, sext, $J=$ $7.32 \mathrm{~Hz}), 1.46(2 \mathrm{H}$, quint, $J=7.32 \mathrm{~Hz}), 2.21(3 \mathrm{H}, \mathrm{s}), 2.33(2 \mathrm{H}$, $\mathrm{t}, J=7.32 \mathrm{~Hz}), 3.02(2 \mathrm{H}, \mathrm{d}, J=5.86 \mathrm{~Hz}), 3.12(2 \mathrm{H}, \mathrm{d}, J=$ $6.34 \mathrm{~Hz}), 4.15(2 \mathrm{H}, \mathrm{q}, J=7.07 \mathrm{~Hz}), 5.68(1 \mathrm{H}, \mathrm{dt}, J=10.73$, $6.34 \mathrm{~Hz}), 5.74(1 \mathrm{H}, \mathrm{dt}, J=10.73,5.86 \mathrm{~Hz}) .{ }^{1} \mathrm{H} \mathrm{NMR}\left(\mathrm{CDCl}_{3}\right.$ with $\left.\mathrm{CF}_{3} \mathrm{CO}_{2} \mathrm{D}\right) \delta 3.10-3.25(4 \mathrm{H}, \mathrm{m})$. E isomer; ${ }^{1} \mathrm{HNMR}\left(\mathrm{CDCl}_{3}\right) \delta$ $0.91(3 \mathrm{H}, \mathrm{t}, J=7.32 \mathrm{~Hz}), 1.26(3 \mathrm{H}, \mathrm{t}, J=7.07 \mathrm{~Hz}), 1.31(2 \mathrm{H}$, sext, $J=7.32 \mathrm{~Hz}), 1.45(2 \mathrm{H}$, quint, $J=7.32 \mathrm{~Hz}), 2.21(3 \mathrm{H}, \mathrm{s})$, $2.34(2 \mathrm{H}, \mathrm{t}, J=7.32 \mathrm{~Hz}), 2.99(2 \mathrm{H}, \mathrm{d}, J=6.59 \mathrm{~Hz}), 3.07(2 \mathrm{H}$, $\mathrm{d}, J=6.34 \mathrm{~Hz}), 4.14(2 \mathrm{H}, \mathrm{q}, J=7.07 \mathrm{~Hz}), 5.63(1 \mathrm{H}, \mathrm{dt}$, $J=15.86,6.34 \mathrm{~Hz}), 5.71(1 \mathrm{H}, \mathrm{dt}, J=15.86,6.59 \mathrm{~Hz}) .{ }^{1} \mathrm{HNMR}$ $\left(\mathrm{CDCl}_{3}\right.$ with $\left.\mathrm{CF}_{3} \mathrm{CO}_{2} \mathrm{D}\right) \delta$ 2.95-3.05 $(4 \mathrm{H}, \mathrm{m})$. HRMS $\left(\mathrm{FAB}^{+}\right)$ $(\mathrm{M}+\mathrm{H})^{+}$, Found: $m / z$ 214.18101. Calcd for $\mathrm{C}_{12} \mathrm{H}_{24} \mathrm{NO}_{2}$ : 214.18070.

Ethyl 5-(Isopropylmethylamino)-3-pentenoate (5i): An oil $(\mathrm{Z} / \mathrm{E}=82 / 18)$. IR (neat) 3030, 2966, 2930, 2880, 2840, 2789, $1739,1652,1456,1366,1320,1258,1176,1094,1034,974$, $874,796,688 \mathrm{~cm}^{-1} . \mathrm{Z}$ isomer; ${ }^{1} \mathrm{HNMR}\left(\mathrm{CDCl}_{3}\right) \delta 1.05(6 \mathrm{H}, \mathrm{d}$, $J=6.59 \mathrm{~Hz}), 1.26(3 \mathrm{H}, \mathrm{t}, J=7.07 \mathrm{~Hz}), 2.21(3 \mathrm{H}, \mathrm{s}), 2.90(1 \mathrm{H}$, sept, $J=6.59 \mathrm{~Hz}), 3.10(2 \mathrm{H}, \mathrm{d}, J=6.10 \mathrm{~Hz}), 3.13(2 \mathrm{H}, \mathrm{d}, J=$ $5.85 \mathrm{~Hz}), 4.15(2 \mathrm{H}, \mathrm{q}, J=7.07 \mathrm{~Hz}), 5.70(1 \mathrm{H}, \mathrm{dt}, J=10.98$, $6.10 \mathrm{~Hz}), 5.74(1 \mathrm{H}, \mathrm{dt}, J=10.98,5.85 \mathrm{~Hz}) .{ }^{1} \mathrm{HNMR}\left(\mathrm{CDCl}_{3}\right.$ with 

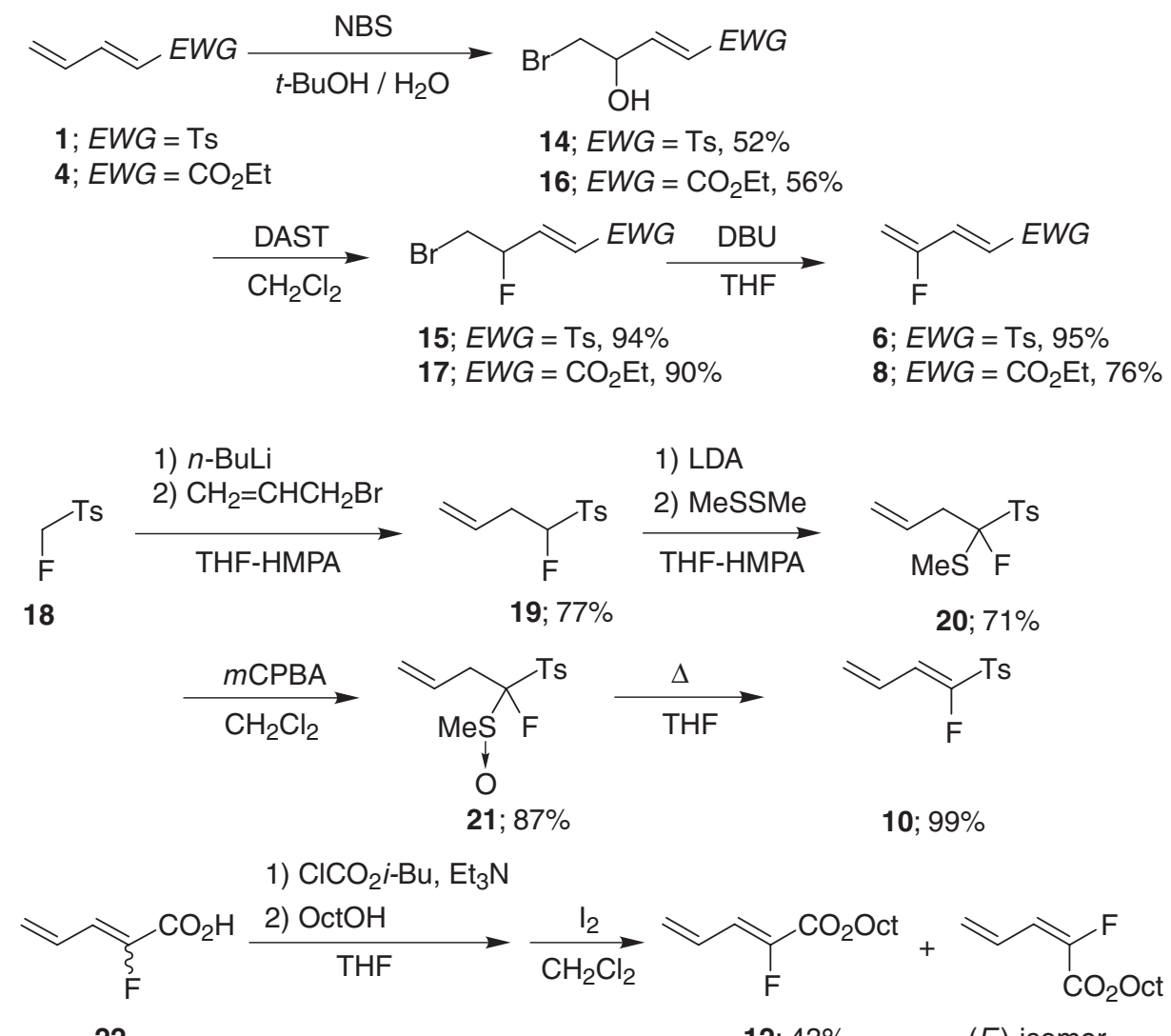

Scheme 2.

$\left.\mathrm{CF}_{3} \mathrm{CO}_{2} \mathrm{D}\right) \delta 2.75(3 \mathrm{H}, \mathrm{s}) . \mathrm{E}$ isomer; ${ }^{1} \mathrm{H} \mathrm{NMR}\left(\mathrm{CDCl}_{3}\right) \delta 1.06$ $(6 \mathrm{H}, \mathrm{d}, J=6.59 \mathrm{~Hz}), 1.26(3 \mathrm{H}, \mathrm{t}, J=7.07 \mathrm{~Hz}), 2.23(3 \mathrm{H}, \mathrm{s})$, $2.91(1 \mathrm{H}$, sept, $J=6.59 \mathrm{~Hz}), 3.08(2 \mathrm{H}, \mathrm{d}, J=6.83 \mathrm{~Hz}), 3.10$ $(2 \mathrm{H}, \mathrm{d}, J=6.10 \mathrm{~Hz}), 4.14(2 \mathrm{H}, \mathrm{q}, J=7.07 \mathrm{~Hz}), 5.67(1 \mathrm{H}, \mathrm{dt}$, $J=15.37,6.83 \mathrm{~Hz}), 5.71(1 \mathrm{H}, \mathrm{dt}, J=15.37,6.10 \mathrm{~Hz}) .{ }^{1} \mathrm{H} \mathrm{NMR}$ $\left(\mathrm{CDCl}_{3}\right.$ with $\left.\mathrm{CF}_{3} \mathrm{CO}_{2} \mathrm{D}\right) \quad \delta \quad 2.72\left(3 \mathrm{H}\right.$, s). HRMS $\left(\mathrm{FAB}^{+}\right)$ $(\mathrm{M}+\mathrm{H})^{+}$, Found: $m / z$ 200.16542. Calcd for $\mathrm{C}_{11} \mathrm{H}_{22} \mathrm{NO}_{2}$ : 200.16505 .

Ethyl 5-(Pyrrolidin-1-yl)-3-pentenoate (5j): An oil (Z/E = 59/41). IR (neat) 3029, 2967, 2908, 2875, 2787, 1739, 1655, $1461,1445,1407,1368,1345,1320,1256,1173,1096,1033$, 940, 902, 879, $858 \mathrm{~cm}^{-1} . \mathrm{Z}$ isomer; ${ }^{1} \mathrm{HNMR}\left(\mathrm{CDCl}_{3}\right) \delta 1.26$ $(3 \mathrm{H}, \mathrm{t}, J=7.07 \mathrm{~Hz}), 1.72-1.82(4 \mathrm{H}, \mathrm{m}), 2.50-2.57(4 \mathrm{H}, \mathrm{m})$, $3.14(2 \mathrm{H}, \mathrm{d}, J=6.34 \mathrm{~Hz}), 3.15(2 \mathrm{H}, \mathrm{d}, J=5.86 \mathrm{~Hz}), 4.15(2 \mathrm{H}$, q, $J=7.07 \mathrm{~Hz}), 5.70(1 \mathrm{H}, \mathrm{dt}, J=10.98,6.34 \mathrm{~Hz}), 5.73(1 \mathrm{H}, \mathrm{dt}$, $J=10.98,5.86 \mathrm{~Hz}) .{ }^{1} \mathrm{HNMR}\left(\mathrm{CDCl}_{3}\right.$ with $\left.\mathrm{CF}_{3} \mathrm{CO}_{2} \mathrm{D}\right) \delta 3.73$ $(2 \mathrm{H}, \mathrm{d}, J=5.86 \mathrm{~Hz}) . \mathrm{E}$ isomer; ${ }^{1} \mathrm{H} \mathrm{NMR}\left(\mathrm{CDCl}_{3}\right) \delta 1.26(3 \mathrm{H}, \mathrm{t}$, $J=7.07 \mathrm{~Hz}), 1.72-1.82(4 \mathrm{H}, \mathrm{m}), 2.50-2.57(4 \mathrm{H}, \mathrm{m}), 3.06(2 \mathrm{H}$, $\mathrm{d}, J=5.61 \mathrm{~Hz}), 3.10(2 \mathrm{H}, \mathrm{d}, J=5.37 \mathrm{~Hz}), 4.15(2 \mathrm{H}, \mathrm{q}$, $J=7.07 \mathrm{~Hz}), 5.69(1 \mathrm{H}, \mathrm{dt}, J=15.39,5.61 \mathrm{~Hz}), 5.75(1 \mathrm{H}, \mathrm{dt}$, $J=15.39,5.37 \mathrm{~Hz}) .{ }^{1} \mathrm{HNMR}\left(\mathrm{CDCl}_{3}\right.$ with $\left.\mathrm{CF}_{3} \mathrm{CO}_{2} \mathrm{D}\right) \delta 3.66$ $(2 \mathrm{H}, \mathrm{d}, J=5.37 \mathrm{~Hz})$. HRMS $\left(\mathrm{FAB}^{+}\right)(\mathrm{M}+\mathrm{H})^{+}$, Found: $m / z$ 198.14951. Calcd for $\mathrm{C}_{11} \mathrm{H}_{20} \mathrm{NO}_{2}:$ 198.14940.

Ethyl 5-(Piperidin-1-yl)-3-pentenoate (5k): An oil (Z/E = 68/32). IR (neat) 3030, 2935, 2854, 2790, 1739, 1651, 1455, $1367,1319,1254,1160,1115,1037,999,861,792 \mathrm{~cm}^{-1} . \mathrm{Z}$ isomre; ${ }^{1} \mathrm{HNMR}\left(\mathrm{CDCl}_{3}\right) \delta 1.26(3 \mathrm{H}, \mathrm{t}, J=7.08 \mathrm{~Hz}), 1.38-1.50$ $(2 \mathrm{H}, \mathrm{m}), 1.59$ ( $4 \mathrm{H}$, quint, $J=5.61 \mathrm{~Hz}), 2.31-2.44(4 \mathrm{H}, \mathrm{m}), 2.98$ $(2 \mathrm{H}, \mathrm{d}, J=5.12 \mathrm{~Hz}), 3.12(2 \mathrm{H}, \mathrm{d}, J=5.37 \mathrm{~Hz}), 4.14(2 \mathrm{H}, \mathrm{q}$, $J=7.08 \mathrm{~Hz}), 5.70(1 \mathrm{H}, \mathrm{dt}, J=10.25,5.37 \mathrm{~Hz}), 5.72(1 \mathrm{H}, \mathrm{dt}$,
$J=10.25,5.12 \mathrm{~Hz}) . \mathrm{E}$ isomer; ${ }^{1} \mathrm{HNMR}\left(\mathrm{CDCl}_{3}\right) \delta 1.26(3 \mathrm{H}, \mathrm{t}$, $J=7.08 \mathrm{~Hz}), 1.38-1.50(2 \mathrm{H}, \mathrm{m}), 1.59(4 \mathrm{H}$, quint, $J=5.61 \mathrm{~Hz})$, 2.31-2.44 (4H, m), $2.95(2 \mathrm{H}, \mathrm{d}, J=5.37 \mathrm{~Hz}), 3.07(2 \mathrm{H}, \mathrm{d}$, $J=5.61 \mathrm{~Hz}), 4.13(2 \mathrm{H}, \mathrm{q}, J=7.08 \mathrm{~Hz}), 5.65(1 \mathrm{H}, \mathrm{dt}, J=$ $15.39,5.61 \mathrm{~Hz}), 5.68(1 \mathrm{H}, \mathrm{dt}, J=15.39,5.37 \mathrm{~Hz})$. HRMS $\left(\mathrm{FAB}^{+}\right)(\mathrm{M}+\mathrm{H})^{+}$, Found: $m / z$ 212.16534. Calcd for $\mathrm{C}_{12} \mathrm{H}_{22} \mathrm{NO}_{2}$ : 212.16505 .

Nucleophilic Addition Reaction of Piperidine (2k) to Ethyl (E)-2,4-Pentadienoate (4) under Dilute Conditions at $60^{\circ} \mathrm{C}$ (Table 6, Entry 8). To a solution of ethyl (E)-2,4-pentadienoate (4) $(65 \mathrm{mg}, 0.52 \mathrm{mmol})$ in THF $(83 \mathrm{~mL})$ was added piperidine $(\mathbf{2 k})$ $(0.306 \mathrm{~mL}, 3.09 \mathrm{mmol})$ at $60^{\circ} \mathrm{C}$ under a nitrogen atmosphere. After stirring for $72 \mathrm{~h}$, the reaction mixture was quenched by adding silica gel $(3.02 \mathrm{~g})$, and the solvent was evaporated. The residue was purified by column chromatography $\left(\mathrm{SiO}_{2}\right.$, hexane $/$ EtOAc $=$ $5 / 1, \mathrm{v} / \mathrm{v}, \mathrm{EtOAc}$, then EtOAc $/ \mathrm{MeOH}=3 / 1, \mathrm{v} / \mathrm{v}$ ) to give a mixture of $(Z)$ - and $(E)-5 \mathbf{k}(54 \mathrm{mg}, 50 \%, \mathrm{Z} / \mathrm{E}=89 / 11)$ and to recover unreacted 4 (26 mg, 40\%).

Fluorine-substituted dienes $\mathbf{6}, \mathbf{8}, \mathbf{1 0}$, and 12 were prepared as shown in Scheme 2.

(E)-1-Bromo-4-tosylbut-3-en-2-ol (14): To a solution of 1 (198 mg, $0.951 \mathrm{mmol})$ in $t$-BuOH $(10 \mathrm{~mL})$ and $\mathrm{H}_{2} \mathrm{O}(12 \mathrm{~mL})$ was added NBS ( $254 \mathrm{mg}, 1.43 \mathrm{mmol}$ ). After $48 \mathrm{~h}$, the reaction mixture was quenched with a saturated solution of $\mathrm{NaHSO}_{3}$, and the solvent was evaporated. The organic substances were extracted with $\mathrm{Et}_{2} \mathrm{O}$, followed by washing with $\mathrm{H}_{2} \mathrm{O}$, brine, and dried over $\mathrm{Na}_{2} \mathrm{SO}_{4}$. After evaporating the solvent, the residue was separated by preparative TLC $\left(\mathrm{SiO}_{2}\right.$, hexane/EtOAc $\left.=5 / 1, \mathrm{v} / \mathrm{v}\right)$ to give 14 in $52 \%$ yield $\left(152 \mathrm{mg}\right.$ ). Mp $119.5-120.0^{\circ} \mathrm{C}$ (from hexane/ EtOAc). IR (KBr) 3481, 3054, 2980, 2940, 2870, 1630, 1596, 
1494, 1399, 1375, 1316, 1303, 1145, 1086, 1044, 1018, 971, 915, 813, 733, 706, $662 \mathrm{~cm}^{-1} .{ }^{1} \mathrm{H}$ NMR $\left(\mathrm{CDCl}_{3}\right) \delta 2.43(3 \mathrm{H}, \mathrm{s}), 3.25$ $(1 \mathrm{H}, \mathrm{d}, J=5.37 \mathrm{~Hz}), 3.43(1 \mathrm{H}, \mathrm{dd}, J=10.49,6.10 \mathrm{~Hz}), 3.53$ $(1 \mathrm{H}, \mathrm{dd}, J=10.49,4.39 \mathrm{~Hz}), 4.58-4.63(1 \mathrm{H}, \mathrm{m}), 6.71(1 \mathrm{H}, \mathrm{dd}$, $J=15.37,2.44 \mathrm{~Hz}), 6.92(1 \mathrm{H}, \mathrm{dd}, J=15.37,3.66 \mathrm{~Hz}), 7.33$ $(2 \mathrm{H}, \mathrm{d}, J=7.81 \mathrm{~Hz}), 7.75(2 \mathrm{H}, \mathrm{d}, J=7.81 \mathrm{~Hz})$. Found: C, 43.34; $\mathrm{H}, 4.24 \%$. Calcd for $\mathrm{C}_{11} \mathrm{H}_{13} \mathrm{O}_{3} \mathrm{SBr}: \mathrm{C}, 43.29 ; \mathrm{H}, 4.29 \%$.

(E)-4-Bromo-3-fluoro-1-tosyl-1-butene (15): To a solution of $\mathbf{1 4}(92 \mathrm{mg}, 0.301 \mathrm{mmol})$ in $\mathrm{CH}_{2} \mathrm{Cl}_{2}(4 \mathrm{~mL})$ was added DAST $(0.060 \mathrm{~mL}, 0.452 \mathrm{mmol})$ at $0{ }^{\circ} \mathrm{C}$ under a nitrogen atmosphere. After $4 \mathrm{~h}$, the reaction mixture was quenched with cool water, and the solvent was evaporated. The organic substances were extracted with EtOAc, followed by washing with $\mathrm{H}_{2} \mathrm{O}$, brine, and dried over $\mathrm{Na}_{2} \mathrm{SO}_{4}$. After evaporating the solvent, the residue was purified by preparative TLC $\left(\mathrm{SiO}_{2}\right.$, hexane/EtOAc $\left.=3 / 1, \mathrm{v} / \mathrm{v}\right)$ to give 15 in $94 \%$ yield $\left(87 \mathrm{mg}\right.$ ). $\mathrm{Mp} 94.5-95.0^{\circ} \mathrm{C}$ (from hexane/ EtOAc). IR (KBr) 3075, 3030, 2970, 2920, 1596, 1422, 1315, 1304, 1280, 1210, 1149, 1086, 1019, 964, 934, 841, 812, 795, 708, 696, $666 \mathrm{~cm}^{-1} .{ }^{1} \mathrm{HNMR}\left(\mathrm{CDCl}_{3}\right) \delta 2.45(3 \mathrm{H}, \mathrm{s}), 3.55(1 \mathrm{H}$, ddd, $J=20.91,11.37,5.50 \mathrm{~Hz}), 3.58(1 \mathrm{H}, \mathrm{ddd}, J=17.79$, $11.37,4.95 \mathrm{~Hz}), 5.37(1 \mathrm{H}$, ddddd, $J=46.95,5.50,4.95,3.30$, $1.83 \mathrm{~Hz}), 6.69(1 \mathrm{H}, \mathrm{dd}, J=15.04,1.83 \mathrm{~Hz}), 6.92(1 \mathrm{H}, \mathrm{ddd}$, $J=20.72,15.04,3.30 \mathrm{~Hz}), 7.36(2 \mathrm{H}, \mathrm{d}, J=7.89 \mathrm{~Hz}), 7.78(2 \mathrm{H}$, $\mathrm{d}, J=7.89 \mathrm{~Hz}) .{ }^{19} \mathrm{FNMR}\left(\mathrm{CDCl}_{3}\right) \delta-182.2(1 \mathrm{~F}, \mathrm{ddt}, J=$ $46.95,17.79,20.91 \mathrm{~Hz}$ ). Found: C, 42.81; H, 3.90\%. Calcd for $\mathrm{C}_{11} \mathrm{H}_{12} \mathrm{O}_{2}$ SFBr: C, 43.01; $\mathrm{H}, 3.94 \%$.

(E)-3-Fluoro-1-tosyl-1,3-butadiene (6): To a solution of $\mathbf{1 5}$ $(129 \mathrm{mg}, 0.420 \mathrm{mmol})$ in THF $(4.0 \mathrm{~mL})$ was added DBU $(0.069 \mathrm{~mL}, 0.462 \mathrm{mmol})$ under a nitrogen atmosphere. After $5 \mathrm{~min}$, the reaction mixture was quenched with a saturated solution of $\mathrm{NH}_{4} \mathrm{Cl}$, and the solvent was evaporated. The organic substances were extracted with EtOAc, followed by washing with $\mathrm{H}_{2} \mathrm{O}$, brine, and dried over $\mathrm{Na}_{2} \mathrm{SO}_{4}$. After evaporating the solvent, the residue was purified by column chromatography $\left(\mathrm{SiO}_{2}\right.$, hexane/ EtOAc $=5 / 1, \mathrm{v} / \mathrm{v})$ to give 6 in $95 \%$ yield $(90 \mathrm{mg})$. Mp $90.3-$ $90.5^{\circ} \mathrm{C}$ (from hexane/EtOAc). IR (KBr) 3060, 1649, 1593, 1312, 1291, 1269, 1205, 1146, 1086, 1018, 971, 950, 885, 844, 831, 816, 705, $670 \mathrm{~cm}^{-1} .{ }^{1} \mathrm{H} \mathrm{NMR}\left(\mathrm{CDCl}_{3}\right) \delta 2.45(3 \mathrm{H}, \mathrm{s}), 4.96$ $(1 \mathrm{H}, \mathrm{dd}, J=45.86,3.17 \mathrm{~Hz}), 5.13(1 \mathrm{H}, \mathrm{dd}, J=14.39,3.17 \mathrm{~Hz})$, $6.66(1 \mathrm{H}, \mathrm{d}, J=14.88 \mathrm{~Hz}), 7.05(1 \mathrm{H}, \mathrm{dd}, J=25.13,14.88 \mathrm{~Hz})$, $7.35(2 \mathrm{H}, \mathrm{d}, J=8.05 \mathrm{~Hz}), 7.78(2 \mathrm{H}, \mathrm{d}, J=8.05 \mathrm{~Hz}) .{ }^{19} \mathrm{~F} \mathrm{NMR}$ $\left(\mathrm{CDCl}_{3}\right) \delta-111.8(1 \mathrm{~F}, \mathrm{ddd}, J=45.86,25.13,14.39 \mathrm{~Hz})$. Found: C, 58.68; H, 5.00\%. Calcd for $\mathrm{C}_{11} \mathrm{H}_{11} \mathrm{FO}_{2} \mathrm{~S}$ : C, 58.39; $\mathrm{H}, 4.90 \%$.

Ethyl (E)-5-Bromo-4-hydroxy-2-pentenoate (16): To a solution of $4(252 \mathrm{mg}, 2.00 \mathrm{mmol})$ in $t-\mathrm{BuOH}(5.0 \mathrm{~mL})$ and $\mathrm{H}_{2} \mathrm{O}$ $(6.0 \mathrm{~mL})$ was added NBS $(534 \mathrm{~g}, 3.00 \mathrm{mmol})$. After $20 \mathrm{~h}$, the reaction mixture was quenched with a saturated solution of $\mathrm{NaHSO}_{3}$, and the solvent was evaporated. The organic substances were extracted with $\mathrm{Et}_{2} \mathrm{O}$, followed by washing with $\mathrm{H}_{2} \mathrm{O}$, brine, and dried over $\mathrm{Na}_{2} \mathrm{SO}_{4}$. After evaporating the solvent, the residue was purified by preparative TLC $\left(\mathrm{SiO}_{2}\right.$, hexane/EtOAc $=3 / 1$, $\mathrm{v} / \mathrm{v})$ to give 16 in $56 \%$ yield $(252 \mathrm{mg}$ ) as an oil. IR (neat) 3443 , 2982, 2939, 2904, 2874, 1716, 1659, 1467, 1445, 1420, 1393, 1370, 1307, 1276, 1220, 1182, 1096, 1040, 982, 900, 877, 858, $722,655 \mathrm{~cm}^{-1} .{ }^{1} \mathrm{HNMR}\left(\mathrm{CDCl}_{3}\right) \delta 1.30(3 \mathrm{H}, \mathrm{t}, J=7.08 \mathrm{~Hz})$, $3.15(1 \mathrm{H}, \mathrm{brs}), 3.44(1 \mathrm{H}, \mathrm{dd}, J=10.49,6.59 \mathrm{~Hz}), 3.56(1 \mathrm{H}, \mathrm{dd}$, $J=10.49,4.15 \mathrm{~Hz}), 4.21(2 \mathrm{H}, \mathrm{q}, J=7.08 \mathrm{~Hz}), 4.52-4.60(1 \mathrm{H}$, $\mathrm{m}), 6.16(1 \mathrm{H}, \mathrm{dd}, J=15.61,1.71 \mathrm{~Hz}), 6.90(1 \mathrm{H}, \mathrm{dd}, J=15.61$, $4.65 \mathrm{~Hz})$. HRMS $\left(\mathrm{FAB}^{+}\right)(\mathrm{M}+\mathrm{H})^{+}$, Found: $m / z$ 222.99672, 224.99452. Calcd for $\mathrm{C}_{7} \mathrm{H}_{12} \mathrm{O}_{3}{ }^{79} \mathrm{Br}$ : 222.99698, Calcd for $\mathrm{C}_{7} \mathrm{H}_{12} \mathrm{O}_{3}{ }^{81} \mathrm{Br}$ : 224.99494.

Ethyl (E)-5-Bromo-4-fluoro-2-pentenoate (17): To a solu- tion of $16(125 \mathrm{mg}, 0.560 \mathrm{mmol})$ in $\mathrm{CH}_{2} \mathrm{Cl}_{2}(4.0 \mathrm{~mL})$ was added DAST $(0.111 \mathrm{~mL}, 0.841 \mathrm{mmol})$ at $0{ }^{\circ} \mathrm{C}$ under a nitrogen atmosphere. After $70 \mathrm{~min}$, the reaction mixture was quenched with cool water, and the solvent was evaporated. The organic substances were extracted with EtOAc, followed by washing with $\mathrm{H}_{2} \mathrm{O}$, brine, and dried over $\mathrm{Na}_{2} \mathrm{SO}_{4}$. After evaporating the solvent, the residue was purified by preparative TLC $\left(\mathrm{SiO}_{2}\right.$, hexane/EtOAc $=5 / 1$, $\mathrm{v} / \mathrm{v})$ to give $\mathbf{1 7}$ in $90 \%$ yield $(114 \mathrm{mg}$ ) as an oil. IR (neat) 2983 , 2940, 2906, 2875, 1722, 1665, 1467, 1446, 1420, 1392, 1370, 1347, 1308, 1274, 1183, 1095, 1065, 1044, 979, 902, 876, 857, $825,720,687 \mathrm{~cm}^{-1} .{ }^{1} \mathrm{HNMR}\left(\mathrm{CDCl}_{3}\right) \delta 1.31(3 \mathrm{H}, \mathrm{t}, J=7.07$ $\mathrm{Hz}), 3.51(1 \mathrm{H}, \mathrm{ddd}, J=24.15,9.27,5.86 \mathrm{~Hz}), 3.54(1 \mathrm{H}$, ddd, $J=24.15,9.27,5.86 \mathrm{~Hz}), 4.23(2 \mathrm{H}, \mathrm{q}, J=7.07 \mathrm{~Hz}), 5.29(1 \mathrm{H}$, dddt, $J=47.08,4.39,1.71,5.86 \mathrm{~Hz}), 6.17(1 \mathrm{H}, \mathrm{dt}, J=15.61$, $1.71 \mathrm{~Hz}), 6.88(1 \mathrm{H}$, ddd, $J=19.27,15.61,4.39 \mathrm{~Hz}) .{ }^{19} \mathrm{FNMR}$ $\left(\mathrm{CDCl}_{3}\right) \delta-181.1$ (1F, ddt, $\left.J=47.08,19.27,24.15 \mathrm{~Hz}\right)$. HRMS $\left(\mathrm{FAB}^{+}\right)(\mathrm{M}+\mathrm{H})^{+}$, Found: $m / z$ 224.99253, 226.99238. Calcd for $\mathrm{C}_{7} \mathrm{H}_{11} \mathrm{O}_{2} \mathrm{~F}^{79} \mathrm{Br}$ : 224.99264. Calcd for $\mathrm{C}_{7} \mathrm{H}_{11} \mathrm{O}_{2} \mathrm{FB}^{81} \mathrm{Br}$ : 226.99060 .

Ethyl $(\boldsymbol{E})$-4-Fluoro-2,4-pentadienoate (8): To a solution of $17(76 \mathrm{mg}, 0.338 \mathrm{mmol})$ in THF $(3.0 \mathrm{~mL})$ was added DBU $(0.056 \mathrm{~mL}, 0.371 \mathrm{mmol})$ under a nitrogen atmosphere. After $5 \mathrm{~min}$, the reaction mixture was quenched with a saturated solution of $\mathrm{NH}_{4} \mathrm{Cl}$, and the solvent was evaporated. The organic substances were extracted with EtOAc, followed by washing with $\mathrm{H}_{2} \mathrm{O}$, brine, and dried over $\mathrm{Na}_{2} \mathrm{SO}_{4}$. After evaporating the solvent, the residue was separated by column chromatography $\left(\mathrm{SiO}_{2}\right.$, hexane/ EtOAc $=5 / 1, \mathrm{v} / \mathrm{v})$ to give $\mathbf{8}$ in $76 \%$ yield $(37 \mathrm{mg})$ as an oil. IR (neat) 3050, 2984, 2940, 2906, 1719, 1653, 1618, 1467, 1447, 1395, 1367, 1307, 1258, 1230, 1177, 1096, 1036, 973, 953, 891, 866, $737 \mathrm{~cm}^{-1} .{ }^{1} \mathrm{HNMR}\left(\mathrm{CDCl}_{3}\right) \delta 1.31(3 \mathrm{H}, \mathrm{t}, J=7.08 \mathrm{~Hz})$, $4.23(2 \mathrm{H}, \mathrm{q}, J=7.08 \mathrm{~Hz}), 4.84(1 \mathrm{H}, \mathrm{dd}, J=46.35,2.93 \mathrm{~Hz})$, $5.04(1 \mathrm{H}, \mathrm{dd}, J=14.64,2.93 \mathrm{~Hz}), 6.20(1 \mathrm{H}, \mathrm{d}, J=15.61 \mathrm{~Hz})$, $7.05(1 \mathrm{H}, \quad \mathrm{dd}, \quad J=26.10,15.61 \mathrm{~Hz}) .{ }^{19} \mathrm{FNMR}\left(\mathrm{CDCl}_{3}\right) \quad \delta$ $-112.99(1 \mathrm{~F}, \mathrm{ddd}, J=46.35,26.10,14.64 \mathrm{~Hz})$. HRMS $\left(\mathrm{FAB}^{+}\right)$ $(\mathrm{M}+\mathrm{H})^{+}$, Found: $m / z$ 145.06616. Calcd for $\mathrm{C}_{7} \mathrm{H}_{10} \mathrm{O}_{2} \mathrm{~F}$ : 145.06648 .

4-Fluoro-4-tosyl-1-butene (19): To a mixed solution of $\mathbf{1 8}^{5 \mathrm{~b}}$ (300 mg, $1.59 \mathrm{mmol})$ and HMPA $(0.415 \mathrm{~mL}, 2.39 \mathrm{mmol})$ in THF $(15.9 \mathrm{~mL})$ was added a solution of $n$-BuLi in hexane $(1.14 \mathrm{~mL}$, $1.75 \mathrm{mmol}, 1.54 \mathrm{M})$ at $-72{ }^{\circ} \mathrm{C}$ under a nitrogen atmosphere, followed by addition of allyl bromide $(0.165 \mathrm{~mL}, 1.91 \mathrm{mmol})$ after $30 \mathrm{~min}$. The reaction mixture was stirred for $10 \mathrm{~min}$ at $-72{ }^{\circ} \mathrm{C}$ and for $3 \mathrm{~h}$ at room temperature, and then quenched by addition of a saturated $\mathrm{NH}_{4} \mathrm{Cl}$ solution. After removing the solvent under reduced pressure, the organic substances were extracted with EtOAc, followed by washing with $\mathrm{H}_{2} \mathrm{O}$, brine, and drying over $\mathrm{Na}_{2} \mathrm{SO}_{4}$. After evaporating the solvent, the residue was separated by preparative TLC $\left(\mathrm{SiO}_{2}\right.$, hexane/EtOAc $\left.=5 / 1, \mathrm{v} / \mathrm{v}\right)$ to give 19 in $77 \%$ yield $(278 \mathrm{mg}$ ) as an oil. IR (neat) 3084, 2983, 2925, $1644,1597,1494,1431,1375,1330,1305,1245,1221,1185$, 1155, 1090, 1078, 1044, 1018, 989, 928, 867, 816, 737, 705, $663 \mathrm{~cm}^{-1} .{ }^{1} \mathrm{HNMR}\left(\mathrm{CDCl}_{3}\right) \delta 2.47(3 \mathrm{H}, \mathrm{s}), 2.63(1 \mathrm{H}, \mathrm{ddddt}, J=$ 20.00, 15.37, 9.76, 6.83, $1.22 \mathrm{~Hz}), 2.88(1 \mathrm{H}$, ddddt, $J=36.10$, 15.37, 6.83, 2.93, $1.22 \mathrm{~Hz}), 5.10(1 \mathrm{H}, \mathrm{ddd}, J=48.30,9.76$, $2.93 \mathrm{~Hz}), 5.22(1 \mathrm{H}, \mathrm{dd}, J=10.02,1.22 \mathrm{~Hz}), 5.24(1 \mathrm{H}, \mathrm{dd}, J=$ $17.08,1.22 \mathrm{~Hz}), 5.80(1 \mathrm{H}, \mathrm{ddt}, J=17.08,10.02,6.83 \mathrm{~Hz}), 7.39$ $(2 \mathrm{H}, \quad \mathrm{d}, \quad J=8.29 \mathrm{~Hz}), 7.82(2 \mathrm{H}, \quad \mathrm{d}, \quad J=8.29 \mathrm{~Hz}) .{ }^{19} \mathrm{FNMR}$ $\left(\mathrm{CDCl}_{3}\right) \delta-179.7(1 \mathrm{~F}, \mathrm{ddd}, J=48.30,36.10,20.00 \mathrm{~Hz})$. HRMS $\left(\mathrm{FAB}^{+}\right) \quad(\mathrm{M}+\mathrm{H})^{+}$, Found: $m / z$ 229.06941. Calcd for $\mathrm{C}_{11} \mathrm{H}_{14} \mathrm{O}_{2}$ FS: 229.06985 .

(E)-4-Fluoro-4-methylsulfanyl-4-tosyl-1-butene (20): In a 
dry flask, $n$-BuLi in hexane $(0.740 \mathrm{~mL}, 1.14 \mathrm{mmol}, 1.54 \mathrm{M})$ was added to a solution of $i$ - $\mathrm{Pr}_{2} \mathrm{NH}(115 \mathrm{~mL}, 1.14 \mathrm{mmol})$ in THF $(9.4 \mathrm{~mL})$ at $-72{ }^{\circ} \mathrm{C}$ and the mixture was stirred for $30 \mathrm{~min}$, followed by dropwise addition of a solution of $19(220 \mathrm{mg}$, $0.964 \mathrm{mmol})$ in THF $(2.0 \mathrm{~mL})$. After stirring for $30 \mathrm{~min}$ at $-72{ }^{\circ} \mathrm{C}$, MeSSMe $(0.154 \mathrm{~mL}, 1.71 \mathrm{mmol})$ was added to the reaction mixture and the mixture was stirred for $90 \mathrm{~min}$ at room temperature. Then, the reaction mixture was quenched with phosphatebuffer ( $\mathrm{pH}$ 7.2). After evaporating the organic solvent, the product was extracted with EtOAc, followed by washing with $\mathrm{H}_{2} \mathrm{O}$, brine, and drying over $\mathrm{Na}_{2} \mathrm{SO}_{4}$. After evaporating the solvent, the residue was separated by preparative TLC (hexane/EtOAc $=9 / 1$, $\mathrm{v} / \mathrm{v})$ to afford 20 in $71 \%$ yield $(188 \mathrm{mg})$ as an oil. IR (neat) 3083, 3027, 2983, 2935, 1642, 1597, 1493, 1428, 1333, 1306, 1292, 1244, 1212, 1185, 1155, 1087, 1040, 1018, 984, 970, 928, $873,815,762,706 \mathrm{~cm}^{-1}$. ${ }^{1} \mathrm{HNMR}\left(\mathrm{CDCl}_{3}\right) \delta 2.34(3 \mathrm{H}, \mathrm{d}$, $J=1.46 \mathrm{~Hz}), 2.48(3 \mathrm{H}, \mathrm{s}), 2.73-2.81(2 \mathrm{H}, \mathrm{m}), 5.14(1 \mathrm{H}, \mathrm{dd}$, $J=17.08,1.46 \mathrm{~Hz}), 5.21(1 \mathrm{H}, \mathrm{dd}, J=10.00,1.46 \mathrm{~Hz}), 5.79$ $(1 \mathrm{H}, \mathrm{ddt}, J=17.08,10.00,6.83 \mathrm{~Hz}), 7.39(2 \mathrm{H}, \mathrm{d}, J=8.05 \mathrm{~Hz})$, $7.85(2 \mathrm{H}, \mathrm{d}, J=8.05 \mathrm{~Hz}) .{ }^{19} \mathrm{FNMR}\left(\mathrm{CDCl}_{3}\right) \delta-141.3(1 \mathrm{~F}, \mathrm{dd}$, $J=20.65,18.36 \mathrm{~Hz})$. HRMS $\left(\mathrm{FAB}^{+}\right)(\mathrm{M}+\mathrm{H})^{+}$, Found: $m / z$ 275.05846. Calcd for $\mathrm{C}_{12} \mathrm{H}_{16} \mathrm{O}_{2} \mathrm{FS}_{2}: 275.05758$.

(E)-1-Fluoro-1-tosyl-1,3-butadiene (10): Compound 20 $(156 \mathrm{mg}, 0.569 \mathrm{mmol})$ was treated with $m \mathrm{CPBA}$ (ca. $65 \%$, $151 \mathrm{mg}, 0.569 \mathrm{mmol})$ in the presence of $\mathrm{NaHCO}_{3}(96 \mathrm{mg}$, $1.14 \mathrm{mmol})$ in $\mathrm{CH}_{2} \mathrm{Cl}_{2}(6.0 \mathrm{~mL})$ for $30 \mathrm{~min}$ at room temperature. Then, the reaction mixture was quenched with a saturated aqueous solution of $\mathrm{NaHSO}_{3}$. After evaporating the organic solvent, the product was extracted with EtOAc, followed by washing with a saturated solution of $\mathrm{NaHCO}_{3}, \mathrm{H}_{2} \mathrm{O}$, brine, and drying over $\mathrm{Na}_{2} \mathrm{SO}_{4}$. After removal of solvent, the residue was purified by preparative TLC (hexane/EtOAc $=5 / 1, \mathrm{v} / \mathrm{v}$ ) to afford 21 in $87 \%$ yield $(144 \mathrm{mg})$. A THF ( $4.0 \mathrm{~mL})$ solution of the obtained sulfoxide $21(65 \mathrm{mg}, 0.224 \mathrm{mmol})$ was refluxed for $2 \mathrm{~h}$. The reaction mixture was condensed in vacuo to afford $\mathbf{1 0}$ in $99 \%$ yield $(50 \mathrm{mg})$ as an oil. The obtained $\mathbf{1 0}$ was used for the reaction with diethylamine without further purification. IR (neat) 3050, 2925, 2854, 1812, $1724,1651,1596,1492,1457,1333,1305,1212,1154,1087$, 984, 930, 815, 706, $666 \mathrm{~cm}^{-1} .{ }^{1} \mathrm{HNMR}\left(\mathrm{CDCl}_{3}\right) \delta 2.46(3 \mathrm{H}, \mathrm{s})$, $5.51(1 \mathrm{H}, \mathrm{d}, J=10.25 \mathrm{~Hz}), 5.65(1 \mathrm{H}, \mathrm{d}, J=17.08 \mathrm{~Hz}), 6.49$ $(1 \mathrm{H}, \mathrm{ddd}, J=17.08,10.98,10.25 \mathrm{~Hz}), 6.70(1 \mathrm{H}, \mathrm{dd}, J=30.74$, $10.98 \mathrm{~Hz}), 7.38(2 \mathrm{H}, \mathrm{d}, J=8.05 \mathrm{~Hz}), 7.83(2 \mathrm{H}, \mathrm{d}, J=8.05 \mathrm{~Hz})$. ${ }^{19} \mathrm{FNMR}\left(\mathrm{CDCl}_{3}\right) \delta-126.9(1 \mathrm{~F}, \mathrm{~d}, \quad J=30.74 \mathrm{~Hz})$. HRMS $\left(\mathrm{FAB}^{+}\right) \quad(\mathrm{M}+\mathrm{H})^{+}$, Found: $m / z$ 227.05495. Calcd for $\mathrm{C}_{11} \mathrm{H}_{12} \mathrm{O}_{2}$ FS: 227.05420 .

Octyl (Z)-2-Fluoro-2,4-pentadienoate (12): $\quad$ To a mixed solution of $(Z)$ - and (E)-2-fluoro-2,4-pentadienoic acid $(\mathbf{2 2})^{23}$ $(252 \mathrm{mg}, 2.17 \mathrm{mmol})$ in THF $(5.0 \mathrm{~mL})$ was added triethylamine $(0.303 \mathrm{~mL}, 2.17 \mathrm{mmol})$ and isobutyl chloroformate $(0.282 \mathrm{~mL}$, $2.17 \mathrm{mmol})$ at $-15^{\circ} \mathrm{C}$ under a nitrogen atmosphere, followed by addition of octanol $(0.684 \mathrm{~mL}, 4.34 \mathrm{mmol})$ after $30 \mathrm{~min}$. The reaction mixture was stirred at room temperature overnight, and then quenched by adding a saturated $\mathrm{NH}_{4} \mathrm{Cl}$ solution, and the solvent was evaporated. The organic substances were extracted with EtOAc, followed by washing with brine, and dried over $\mathrm{Na}_{2} \mathrm{SO}_{4}$. After evaporating the solvent, the residue was purified by preparative TLC $\left(\mathrm{SiO}_{2}\right.$, hexane $\left./ \mathrm{EtOAc}=30 / 1, \mathrm{v} / \mathrm{v}\right)$ to give a mixture of $(Z)$-ester 12 and the corresponding $\mathrm{E}$ isomer. Then, the mixture of esters $(291 \mathrm{mg}, 1.27 \mathrm{mmol})$ was treated with iodide $(49 \mathrm{mg}$, $0.191 \mathrm{mmol})$ in $\mathrm{CH}_{2} \mathrm{Cl}_{2}(5.0 \mathrm{~mL})$ at room temperature overnight to isomerize $(E)$-ester to $(Z)$-ester 12 . To the reaction mixture was added $\mathrm{H}_{2} \mathrm{O}$ and the product was extracted with EtOAc, fol- lowed by washing with a saturated solution of $\mathrm{NaHSO}_{3}$, brine, and drying over $\mathrm{Na}_{2} \mathrm{SO}_{4}$. The solvent was evaporated and the resulting residue was purified by recycle HPLC $\left(\mathrm{SiO}_{2}\right.$, hexane/ EtOAc $=40 / 1, \mathrm{v} / \mathrm{v})$ to give $(Z)$-ester 12 in $43 \%$ yield $(214 \mathrm{mg})$ as an oil. IR (neat) 3094, 2957, 2927, 2857, 1824, 1729, 1646, $1598,1468,1421,1391,1380,1338,1288,1227,1189,1140$, 1100, 1000, 923, 774, 723, 658 cm $\mathrm{cm}^{-1} .{ }^{1} \mathrm{HNMR}\left(\mathrm{CDCl}_{3}\right) \delta 0.89$ $(3 \mathrm{H}, \mathrm{t}, J=7.08 \mathrm{~Hz}), 1.30-1.40(10 \mathrm{H}, \mathrm{m}), 1.70(2 \mathrm{H}$, quint, $J=$ $6.83 \mathrm{~Hz}), 4.23(2 \mathrm{H}, \mathrm{t}, J=6.83 \mathrm{~Hz}), 5.44(1 \mathrm{H}, \mathrm{d}, J=10.25 \mathrm{~Hz})$, $5.58(1 \mathrm{H}, \mathrm{d}, J=16.59 \mathrm{~Hz}), 6.58(1 \mathrm{H}, \mathrm{dd}, J=29.76,10.98 \mathrm{~Hz})$, $6.68(1 \mathrm{H}, \mathrm{ddd}, J=16.59,10.98,10.25 \mathrm{~Hz}) .{ }^{19} \mathrm{~F} \mathrm{NMR}\left(\mathrm{CDCl}_{3}\right)$ $\delta-128.48(1 \mathrm{~F}, \mathrm{~d}, J=29.78 \mathrm{~Hz}) . \operatorname{HRMS}\left(\mathrm{FAB}^{+}\right)(\mathrm{M}+\mathrm{H})^{+}$, Found: $m / z$ 229.15848. Calcd for $\mathrm{C}_{13} \mathrm{H}_{22} \mathrm{O}_{2} \mathrm{~F}: 229.16038$.

The addition reaction of amines $\mathbf{2 d}$ and $\mathbf{2 k}$ to the synthesized fluorine-substituted dienes $\mathbf{6}, \mathbf{8}, \mathbf{1 0}$, and $\mathbf{1 2}$ was carried out in a similar manner described for the addition to $\mathbf{1}$ and $\mathbf{4}$. The physical and spectral data of the corresponding allylic products are given in the following.

$\boldsymbol{N}, \boldsymbol{N}$-Diethyl-2-fluoro-4-tosyl-2-butenylamine (7): An oil $(Z / E=95 / 5)$. IR (neat) 2970, 2927, 2873, 1705, 1597, 1455, $1385,1318,1303,1147,1087,920,817,753,669 \mathrm{~cm}^{-1} . \mathrm{Z}$ isomer; ${ }^{1} \mathrm{HNMR}\left(\mathrm{CDCl}_{3}\right) \delta 0.96(6 \mathrm{H}, \mathrm{t}, J=7.08 \mathrm{~Hz}), 2.44(3 \mathrm{H}, \mathrm{s}), 2.44$ $(4 \mathrm{H}, \mathrm{q}, J=7.08 \mathrm{~Hz}), 3.08(2 \mathrm{H}, \mathrm{d}, J=14.88 \mathrm{~Hz}), 3.89(2 \mathrm{H}, \mathrm{d}$, $J=7.81 \mathrm{~Hz}), 4.95(1 \mathrm{H}, \mathrm{dt}, J=33.42,7.81 \mathrm{~Hz}), 7.33(2 \mathrm{H}, \mathrm{d}$, $J=8.05 \mathrm{~Hz}), 7.76(2 \mathrm{H}, \mathrm{d}, J=8.05 \mathrm{~Hz}) .{ }^{19} \mathrm{FNMR}\left(\mathrm{CDCl}_{3}\right) \delta$ $-104.9(1 \mathrm{~F}, \quad \mathrm{dt}, \quad J=33.42,14.88 \mathrm{~Hz}) . \quad \mathrm{E}$ isomer; ${ }^{1} \mathrm{HNMR}$ $\left(\mathrm{CDCl}_{3}\right) \delta 0.96(6 \mathrm{H}, \mathrm{t}, J=7.08 \mathrm{~Hz}), 2.44(3 \mathrm{H}, \mathrm{s}), 2.44(4 \mathrm{H}, \mathrm{q}$, $J=7.08 \mathrm{~Hz}), 2.98(2 \mathrm{H}, \mathrm{d}, J=17.81 \mathrm{~Hz}), 3.92(2 \mathrm{H}, \mathrm{d}, J=$ $8.78 \mathrm{~Hz}), 5.22(1 \mathrm{H}, \mathrm{dt}, J=18.78,8.78 \mathrm{~Hz}), 7.33(2 \mathrm{H}, \mathrm{d}, J=$ $8.05 \mathrm{~Hz}), 7.76(2 \mathrm{H}, \mathrm{d}, J=8.05 \mathrm{~Hz}) .{ }^{19} \mathrm{FNMR}\left(\mathrm{CDCl}_{3}\right) \delta-92.0$ $(1 \mathrm{~F}, \mathrm{dt}, J=18.78,17.81 \mathrm{~Hz})$. HRMS $\left(\mathrm{FAB}^{+}\right)(\mathrm{M}+\mathrm{H})^{+}$, Found: $m / z$ 300.14305. Calcd for $\mathrm{C}_{15} \mathrm{H}_{23} \mathrm{NO}_{2} \mathrm{SF}: 300.14335$. The stereochemistry of $(Z)$ - and $(E)-7$ was assigned based on $J_{\mathrm{H}-\mathrm{F}}$ through the double bond. ${ }^{24}$ Furthermore, NOE analysis between methylene protons and a vinylic proton in ( $Z$ ) -7 supported the assignment (Figure 4).

Ethyl 4-Fluoro-5-(piperidin-1-yl)-3-pentenoate (9): An oil $(\mathrm{Z} / \mathrm{E}=99 / 1) . \mathrm{IR}$ (neat) 2970, 2937, 2856, 2807, 1739, 1371, $1343,1308,1248,1183,1029,993,949,864,669 \mathrm{~cm}^{-1} . \mathrm{Z}$ isomer; ${ }^{1} \mathrm{H} \mathrm{NMR}\left(\mathrm{CDCl}_{3}\right) \delta 1.26(3 \mathrm{H}, \mathrm{t}, J=7.07 \mathrm{~Hz}), 1.40-1.48(2 \mathrm{H}, \mathrm{m})$, $1.60(4 \mathrm{H}$, quint, $J=5.61 \mathrm{~Hz}), 2.40-2.48(4 \mathrm{H}, \mathrm{m}), 3.05(2 \mathrm{H}, \mathrm{d}$, $J=18.54 \mathrm{~Hz}), 3.16(2 \mathrm{H}, \mathrm{dd}, J=7.31,0.98 \mathrm{~Hz}), 4.15(2 \mathrm{H}, \mathrm{q}$, $J=7.07 \mathrm{~Hz}), 4.97(1 \mathrm{H}, \mathrm{dt}, J=35.62,7.32 \mathrm{~Hz}) . \quad \mathrm{E}$ isomer; ${ }^{1} \mathrm{HNMR} \quad\left(\mathrm{CDCl}_{3}\right) \quad \delta 1.26 \quad(3 \mathrm{H}, \quad \mathrm{t}, \quad J=7.07 \mathrm{~Hz}), \quad 1.40-1.48$ $(2 \mathrm{H}, \mathrm{m}), 1.60(4 \mathrm{H}$, quint, $J=5.61 \mathrm{~Hz}), 2.40-2.48(4 \mathrm{H}, \mathrm{m}), 2.88$ $(2 \mathrm{H}, \mathrm{d}, J=18.05 \mathrm{~Hz}), 3.32(2 \mathrm{H}, \mathrm{d}, J=8.05 \mathrm{~Hz}), 4.15(2 \mathrm{H}, \mathrm{q}$, $J=7.07 \mathrm{~Hz}), \quad 5.44 \quad(1 \mathrm{H}, \quad \mathrm{dt}, \quad J=20.25, \quad 8.05 \mathrm{~Hz}) .{ }^{19} \mathrm{FNMR}$ $\left(\mathrm{CDCl}_{3}\right) \delta-100.2(1 \mathrm{~F}, \mathrm{dt}, J=20.25,18.05 \mathrm{~Hz})$. HRMS $\left(\mathrm{FAB}^{+}\right)$ $(\mathrm{M}+\mathrm{H})^{+}$, Found: $m / z$ 230.15619. Calcd for $\mathrm{C}_{12} \mathrm{H}_{21} \mathrm{NO}_{2} \mathrm{~F}$ : 230.15563. The stereochemistry of $(Z)$ - and $(E)-9$ was assigned based on $J_{\mathrm{H}-\mathrm{F}}$ through the double bond. ${ }^{24}$

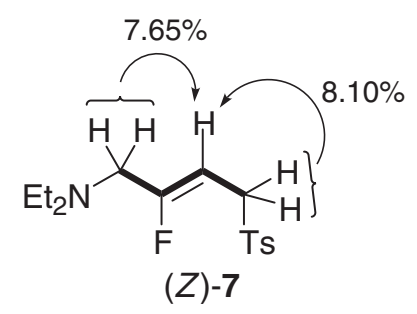

Figure 4. 
$\mathrm{N}, \boldsymbol{N}$-Diethyl-4-fluoro-4-tosyl-2-butenylamine (11): $\quad$ An oil $(\mathrm{Z} / \mathrm{E}=62 / 38)$. IR (neat) 2970, 2933, 2808, 1596, 1492, 1458, $1384,1330,1305,1200,1153,1088,1045,816,712,669 \mathrm{~cm}^{-1}$. $\mathrm{Z}$ isomer; ${ }^{1} \mathrm{HNMR}\left(\mathrm{CDCl}_{3}\right) \delta 1.02(6 \mathrm{H}, \mathrm{t}, J=7.08 \mathrm{~Hz}), 2.48$ $(3 \mathrm{H}, \mathrm{s}), 2.50(4 \mathrm{H}, \mathrm{q}, J=7.08 \mathrm{~Hz}), 3.19-3.23(2 \mathrm{H}, \mathrm{m}), 5.48(1 \mathrm{H}$, ddd, $J=13.91,11.47,8.54 \mathrm{~Hz}), 6.05(1 \mathrm{H}, \mathrm{dd}, J=47.33$, $8.54 \mathrm{~Hz}), 6.18(1 \mathrm{H}, \mathrm{dt}, \quad J=11.47,5.61 \mathrm{~Hz}), 7.39(2 \mathrm{H}, \mathrm{d}$, $J=8.05 \mathrm{~Hz}), 7.81(2 \mathrm{H}, \mathrm{d}, J=8.05 \mathrm{~Hz}) .{ }^{19} \mathrm{FNMR}\left(\mathrm{CDCl}_{3}\right) \delta$ $-171.3(1 \mathrm{~F}, \quad \mathrm{dd}, \quad J=47.33,13.91 \mathrm{~Hz})$. E isomer; ${ }^{1} \mathrm{HNMR}$ $\left(\mathrm{CDCl}_{3}\right) \delta 1.00(6 \mathrm{H}, \mathrm{t}, J=7.08 \mathrm{~Hz}), 2.47(3 \mathrm{H}, \mathrm{s}), 2.47(4 \mathrm{H}, \mathrm{q}$, $J=7.08 \mathrm{~Hz}), 3.11-3.21(2 \mathrm{H}, \mathrm{m}), 5.50(1 \mathrm{H}, \mathrm{dd}, J=47.08$, $6.34 \mathrm{~Hz}), 5.77(1 \mathrm{H}, \mathrm{ddd}, J=15.37,15.12,6.34 \mathrm{~Hz}), 6.10(1 \mathrm{H}$, $\mathrm{dt}, J=15.12,6.10 \mathrm{~Hz}), 7.37(2 \mathrm{H}, \mathrm{d}, J=8.05 \mathrm{~Hz}), 7.79(2 \mathrm{H}, \mathrm{d}$, $J=8.05 \mathrm{~Hz}) .{ }^{19} \mathrm{~F} \mathrm{NMR}\left(\mathrm{CDCl}_{3}\right) \delta-174.7(1 \mathrm{~F}, \mathrm{dd}, J=47.08$, $15.37 \mathrm{~Hz})$. HRMS $\left(\mathrm{FAB}^{+}\right)(\mathrm{M}+\mathrm{H})^{+}$, Found: $m / z \quad 300.14327$. Calcd for $\mathrm{C}_{15} \mathrm{H}_{23} \mathrm{NO}_{2} \mathrm{SF}: 300.14335$.

Octyl 2-Fluoro-5-(piperidin-1-yl)-3-pentenoate (13): An oil $(\mathrm{Z} / \mathrm{E}=50 / 50)$. IR (neat) 2931, 2856, 2799, 2760, 1764, 1742, $1467,1278,1197,1156,1119,1039,991,862 \mathrm{~cm}^{-1} . \mathrm{Z}$ isomer; ${ }^{1} \mathrm{HNMR}\left(\mathrm{CDCl}_{3}\right) \delta 0.88(3 \mathrm{H}, \mathrm{t}, J=6.83 \mathrm{~Hz}), 1.20-1.40(10 \mathrm{H}$, $\mathrm{m}), 1.40-1.50(2 \mathrm{H}, \mathrm{m}), 1.58(4 \mathrm{H}$, quint, $J=5.61 \mathrm{~Hz}), 1.64(2 \mathrm{H}$, quint, $J=6.83 \mathrm{~Hz}), 2.30-2.50(4 \mathrm{H}, \mathrm{m}), 3.10-3.25(2 \mathrm{H}, \mathrm{m}), 4.19$ $(2 \mathrm{H}, \mathrm{dt}, J=6.83,1.46 \mathrm{~Hz}), 5.62(1 \mathrm{H}, \mathrm{ddd}, J=46.59,9.27$, $1.78 \mathrm{~Hz}), 5.68(1 \mathrm{H}, \mathrm{dtt}, J=10.98,9.27,1.46 \mathrm{~Hz}), 5.91(1 \mathrm{H}, \mathrm{dt}$, $J=10.98,6.83 \mathrm{~Hz}) .{ }^{19} \mathrm{FNMR}\left(\mathrm{CDCl}_{3}\right) \delta-182.9(1 \mathrm{~F}, \mathrm{dd}$, $J=46.59,9.27 \mathrm{~Hz}) . \mathrm{E}$ isomer; ${ }^{1} \mathrm{HNMR}\left(\mathrm{CDCl}_{3}\right) \delta 0.88(3 \mathrm{H}, \mathrm{t}$, $J=6.83 \mathrm{~Hz}), 1.20-1.40(10 \mathrm{H}, \mathrm{m}), 1.40-1.50(2 \mathrm{H}, \mathrm{m}), 1.58(4 \mathrm{H}$, quint, $J=5.61 \mathrm{~Hz}), 1.64(2 \mathrm{H}$, quint, $J=6.83 \mathrm{~Hz}), 2.30-2.50$ $(4 \mathrm{H}, \mathrm{m}), 2.98-3.03(2 \mathrm{H}, \mathrm{m}), 4.19(2 \mathrm{H}, \mathrm{dt}, J=1.22,6.83 \mathrm{~Hz})$, $5.26(1 \mathrm{H}, \quad \mathrm{ddd}, \quad J=48.81,6.34,1.78 \mathrm{~Hz}), 5.77(1 \mathrm{H}, \mathrm{dtt}$, $J=6.34,15.37,1.46 \mathrm{~Hz}), 6.08(1 \mathrm{H}, \mathrm{dddt}, J=15.37,3.42,1.22$, $6.59 \mathrm{~Hz}) .{ }^{19} \mathrm{FNMR}\left(\mathrm{CDCl}_{3}\right) \delta-184.8(1 \mathrm{~F}, \mathrm{dd}, \quad J=48.81$, $15.37 \mathrm{~Hz})$. HRMS $\left(\mathrm{FAB}^{+}\right)(\mathrm{M}+\mathrm{H})^{+}$, Found: $m / z$ 314.24791. Calcd for $\mathrm{C}_{18} \mathrm{H}_{33} \mathrm{NO}_{2} \mathrm{~F}: 314.24953$.

The present work was financially supported in part by Grant-in-Aid for Scientific Research from Japan Society for the Promotion of Science (JSPS).

\section{References}

1 N. S. Simpkins, Sulphones in Organic Synthesis, Pergamon Press, Oxford, 1993.

2 K. Inomata, J. Synth. Org. Chem., Jpn. 1992, 50, 326.

3 T. Hirata, Y. Sasada, T. Ohtani, T. Asada, H. Kinoshita, H. Senda, K. Inomata, Bull. Chem. Soc. Jpn. 1992, 65, 75, and references cited therein.

4 The "syn effect" is herein defined as an effect which stabilizes the syn conformation against the steric hindrance at the transition state.

5 Related studies on the "syn effect": a) A. Shibayama, T. Nakamura, T. Asada, T. Shintani, Y. Ukaji, H. Kinoshita, K. Inomata, Bull. Chem. Soc. Jpn. 1997, 70, 381. b) T. Nakamura, S. K. Guha, Y. Ohta, D. Abe, Y. Ukaji, K. Inomata, Bull. Chem. Soc. Jpn. 2002, 75, 2031. c) S. K. Guha, A. Shibayama, D. Abe, Y. Ukaji, K. Inomata, Chem. Lett. 2003, 32, 778. d) S. K. Guha, Y. Ukaji, K. Inomata, Chem. Lett. 2003, 32, 1158. e) S. K. Guha, A. Shibayama, D. Abe, M. Sakaguchi, Y. Ukaji, K. Inomata, Bull. Chem. Soc. Jpn. 2004, 77, 2147. f) H. Takenaka, Y. Ukaji, K. Inomata, Chem. Lett. 2005, 34, 256. g) N. Takeda, T. Chayama, H. Takenaka, Y. Ukaji, K. Inomata, Chem. Lett. 2005, 34, 1140.

6 a) T. G. Back, M. Parvez, J. E. Wulff, J. Org. Chem. 2003,
68, 2223. b) L. Bernardi, J. López-Cantarero, B. Niess, K. A. Jørgensen, J. Am. Chem. Soc. 2007, 129, 5772. c) F. Näf, R. Decorzant, S. D. Escher, Tetrahedron Lett. 1982, 23, 5043.

7 A conjugate addition reaction of piperidine to an $\alpha$-substituted dienyl sulfone, ( $2 E)$-2-tosyl-2,4-pentadiene, produced the corresponding $(E)$-allylic sulfones stereoselectively: T. Cuvigny, C. Herve du Penhoat, M. Julia, Tetrahedron 1986, 42, 5321.

8 Preliminary results have been reported: M. Yamazaki, S. K. Guha, Y. Ukaji, K. Inomata, Chem. Lett. 2006, 35, 514.

9 By ${ }^{1} \mathrm{HNMR}$ measurement of the addition reaction of $\mathrm{Et}_{2} \mathrm{NH}(\mathbf{2 d})(150 \mathrm{mM})$ to $\mathbf{1}$ at $25^{\circ} \mathrm{C}, \mathrm{Z} / \mathrm{E}$ ratios of $\mathbf{3 d}$ were found to be almost constant (between $75 / 25$ and $73 / 27$ ) in a period of $92 \mathrm{~h}$.

10 a) R. Ballini, G. Bosica, D. Fiorini, Tetrahedron Lett. 2001, 42, 8471. b) D. Pettig, U. Schöllkopf, Synthesis 1988, 173. c) U. M. Dzhemilev, A. Z. Yakupova, S. K. Minsker, G. A. Tolstikov, J. Org. Chem. USSR. 1979, 15, 1041. d) E. J. Corey, C. U. Kim, R. H. K. Chen, M. Takeda, J. Am. Chem. Soc. 1972, 94, 4395. e) G. de la Herrán, C. Murcia, A. G. Csákÿ, Org. Lett. 2005, 7, 5629 .

11 In the cases of $\mathbf{5} \mathbf{c}-\mathbf{5} \mathbf{e}$ and $\mathbf{5} \mathbf{g}-\mathbf{5} \mathbf{j}$, the $\mathrm{Z} / \mathrm{E}$ ratios were determined by the spectra measured in $\mathrm{CDCl}_{3}$ containing $\mathrm{CF}_{3} \mathrm{CO}_{2} \mathrm{D}$ to separate ${ }^{1} \mathrm{HNMR}$ peaks, and no isomerization was observed for $24 \mathrm{~h}$. Specific chemical shifts used for determination of $\mathrm{Z} / \mathrm{E}$ ratios, which were measured in $\mathrm{CDCl}_{3}$ containing $\mathrm{CF}_{3} \mathrm{CO}_{2} \mathrm{D}$, are shown in Experimental Section.

12 Reaction conditions: $4(100 \mathrm{mM}), \mathrm{Et}_{2} \mathrm{NH}(300 \mathrm{mM})$, proton source $(150 \mathrm{mM}), \mathrm{THF}, 25^{\circ} \mathrm{C}, 24 \mathrm{~h}$. Without proton source (4\% yield; $\mathrm{Z} / \mathrm{E}=83 / 17), \mathrm{CF}_{3} \mathrm{CO}_{2} \mathrm{H}\left(\mathrm{p} K_{\mathrm{a}}=0 ; 25 \mathrm{a} 42 \% ; 7 / 93\right)$, $\mathrm{HCO}_{2} \mathrm{H}\left(3.7 ;{ }^{25 \mathrm{a}} 14 \% ; 12 / 88\right), 2,4,6$-tri $(t$-butyl)phenol $(12.19 ; 25 \mathrm{c}$ $10 \%$; 66/34), EtOH $\left(17 ;{ }^{25 \mathrm{a}} 6 \% ; 73 / 27\right), i$-PrOH $(18,25 \mathrm{~b} 5 \%$; $82 / 18), t$-BuOH $(19 ; 25 \mathrm{a} 5 \%$; $83 / 17)$.

13 Radical mechanism could be ruled out because the presence of TEMPO, $N$-hydroxyphthalimide, or galvinoxyl free radical did not affect the $\mathrm{Z} / \mathrm{E}$ ratios.

14 Crystal structure of an (E)-1,3-butadienyl sulfone revealed the conformation of its diene moiety to be s-trans: M. Zeller, A. D. Hunter, P. Sampson, N. Chumachenko, Acta Crystallogr., Sect. E 2006, 62, o1369; An X-ray analysis of (E)-2,4-pentandienoic acid also proved to be s-trans conformation: F. A. Cotton, J. P. Donahue, C. A. Murillo, J. Am. Chem. Soc. 2003, 125, 5436;

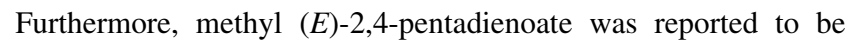
predominantly in the planar s-trans conformation in solution: T. Fueno, K. Yamaguchi, J. Am. Chem. Soc. 1972, 94, 1119.

15 a) S. Winstein, J. Am. Chem. Soc. 1959, 81, 6524. b) P. v. R. Schleyer, J. D. Dill, J. A. Pople, W. J. Hehre, Tetrahedron 1977, 33, 2497. c) L. A. Paquette, Angew. Chem., Int. Ed. Engl. 1978, 17, 106. d) D. Cremer, J. Am. Chem. Soc. 1979, 101, 7199. e) K. N. Houk, R. W. Strozier, N. G. Rondan, R. R. Fraser, N. Chuaqui-Offermanns, J. Am. Chem. Soc. 1980, 102, 1426. f) J. D. Fenske, K. T. Kuwata, K. N. Houk, S. E. Paulson, J. Phys. Chem. A 2000, 104, 7246. g) S. Mangelinckx, N. Giubellina, N. D. Kimpe, Chem. Rev. 2004, 104, 2353, and references cited therein.

16 An effective $\mathrm{n} \rightarrow \pi^{*}$ interaction cannot occur in the conformation $\mathbf{E}$.

17 The order of hyperconjugative electron-donating ability of bonds to carbon has been reported to be $\mathrm{C}-\mathrm{H}>\mathrm{C}-\mathrm{C}>\mathrm{C}-\mathrm{F}$. See: P. R. Rablen, R. W. Hoffmann, D. A. Hrovat, W. T. Borden, J. Chem. Soc., Perkin Trans. 2 1999, 1719.

18 Both linear and cyclic structures were discussed as to amine dimers: a) O. Banjoko, C. Ezeani, J. Chem. Soc., Perkin 
Trans. 2 1986, 531. b) E. T. Akinyele, I. Onyido, J. Hirst, J. Chem. Soc., Perkin Trans. 2 1988, 1859.

19 A related MO study on conjugate addition of amines: E. J. Land, C. A. Ramsden, P. A. Riley, Tetrahedron 2006, 62, 4884.

20 High volatility of ethyl (Z)-2-fluoro-2,4-pentadienoate made purification and handling difficult. Previously, during the investigation of the "syn effect" in the conversion of $\alpha, \beta$-unsaturated $(E)$-esters into the corresponding $\beta, \gamma$-unsaturated esters, we observed almost the same tendency in Z/E selectivity between the ethyl ester and the octyl ester. ${ }^{5 \mathrm{c}, 5 \mathrm{e}}$

21 J. Barluenga, J. M. Martínez-Gallo, C. Nájera, F. J. Fañanás, M. Yus, J. Chem. Soc., Perkin Trans. 1 1987, 2605.

22 J. Rodriguez, B. Waegell, Synthesis 1988, 534.

23 W. H. Johnson, Jr., S. C. Wang, T. M. Stanley, R. M. Czerwinski, J. J. Almrud, G. J. Poelarends, A. G. Murzin, C. P.
Whitman, Biochemistry 2004, 43, 10490.

24 A $J_{\mathrm{H}-\mathrm{F}}$ through a double bond in $\mathrm{Z}$ isomer was larger than that of $\mathrm{E}$ isomer which shows that $\mathrm{H}$ and $\mathrm{F}$ on a double bond have a trans configuration in $\mathrm{Z}$ isomer: T. S. Everett, in Chemistry of Organic Fluorine Compounds II, ed. by M. Hudlicky, A. E. Pavlath, American Chemical Society, Washington, DC, 1995, p. 1042; A recent example of assignment of the stereochemistry of vinyl fluorides: S. Hara, T. Guan, M. Yoshida, Org. Lett. 2006, 8, 2639.

25 a) J. B. Hendrickson, D. J. Cram, G. S. Hammond, Organic Chemistry, 3rd ed., McGraw-Hill, Inc., Kogakusha Co., Ltd., Tokyo, 1970, pp. 304-307. b) H. O. House, Modern Synthetic Reactions, 2nd ed., W. A. Benjamin, Inc., Menlo Park, California, 1972, p. 494. c) M. J. Citra, Chemosphere 1999, 38, 191. 